\title{
Homogeneous Kähler submanifolds in complex projective spaces
}

\author{
By Masaru TAKeuchi
}

(Received January 15, 1977)

\section{Introduction}

A Kähler manifold $(M, g)$ is called homogeneous if the group of automorphisms of $(M, g)$ acts transitively on $M$. The $N$-dimensional complex projective space $P_{N}(C)$ endowed with the Fubini-Study metric of constant holomorphic sectional curvature $c>0$ is denoted by $P_{N}(c)$, and called a Fubini-Study space. In this article, we shall study Kähler immersions, i.e. holomorphic isometric immersions, of homogeneous Kähler manifolds into Fubini-Study spaces.

The most typical example of a homogeneous Kähler manifold is a Fubini-Study space $P_{n}(b)$ itself. It is known that the Fubini-Study spaces $P_{n}(b), b>0$, exhaust the homogeneous Kähler structures on the $n$-dimensional complex projective space $P_{n}(C)$. Thus the set $\mathscr{K}$ of homogeneous Kähler structures on $P_{n}(C)$ corresponds one to one to the cone $\boldsymbol{R}^{+}$of positive real numbers. But the set $\mathscr{K}_{0}$ of homogeneous Kähler structures on $P_{n}(C)$ which admit Kähler immersions into Fubini-Study spaces is a very small subset of $\mathscr{K}$. In fact, Calabi [4] showed that the set $\mathscr{K}_{0}$ corresponds one to one to the semigroup $Z^{+}$of positive integers, more precisely, that $P_{n}(b)$ admits a Kähler immersion into a Fubini-Study space $P_{n}(c)$ if and only if $b=\frac{c}{p}$ for some $p \in Z^{+}$. Explicitly, a Kähler imbedding $f: P_{n}\left(\frac{c}{p}\right)$ $\rightarrow P_{\left(\begin{array}{c}n+p \\ p\end{array}\right)-1}(c)$ is given by the $p$-th Veronese map

$$
f:\left[z_{i}\right]_{0 \leqslant i \leqslant n} \mapsto\left[\sqrt{\frac{p !}{p_{0} ! \cdots p_{n} !}} z_{0}^{p_{0}} \cdots z_{n}^{p_{n}}\right]_{p_{0}+\cdots+p_{n}=p},
$$

where $[*]$ denotes the point of the projective space with the homogeneous coordinates $*$.

We shall show in $\S \S 2$ and 3 that this result of Calabi is generalized for homogeneous Kähler manifolds in the following way: Let $(M, g)$ be a

This work was supported by the Sonderforschungsbereich "Theoretische Mathematik" at the University of Bonn. 
homogeneous Kähler manifold which admits a Kähler immersion $f$ into a Fubini-Study space. Then $M$ is compact and simply connected, and $f$ is an imbedding. The set $\mathscr{K}$ of homogeneous Kähler structures on $M$ corresponds one to one to the quotient $A \backslash c^{+}$of a cone $c^{+}$modulo a finite group $A$ acting on $\mathrm{c}^{+}$. Here $\mathrm{c}^{+}$is an open convex cone of a real vector space $\mathfrak{c}$ with dimension equal to the second Betti number $b_{2}(M)$ of $M$. But the set $\mathscr{K}_{0}$ of homogeneous Kähler structures on $M$ which admit Kähler immersions into Fubini-Study spaces (of constant holomorphic sectional curvatures $c$ ) corresponds one to one to the quotient $A \backslash Z_{\mathrm{c}}^{+}$of a discrete $A$-invariant semigroup $Z_{\mathrm{c}}^{+}$in $\mathfrak{c}^{+}$modulo the group $A$. In particular, the subset $\mathscr{E}_{0}$ of $\mathscr{K}_{0}$ consisting of all Kähler Einstein structures in $\mathscr{K}_{0}$ corresponds one to one to a subsemigroup of $Z_{\mathrm{c}}^{+}$which is isomorphic to $Z^{+}$.

It should be noted that the set $\mathscr{K}_{0}$ (resp. $\mathscr{E}_{0}$ ) corresponds one to one to the set of equivalence classes of full Kähler immersions into Fubini-Study spaces of homogeneous Kähler manifolds (resp. of homogeneous Kähler Einstein manifolds) with the underlying complex manifold $M$, in virtue of the rigidity theorem of Calabi [4].

Furthermore, in $\S 2$ we shall construct explicitly all Kähler immersions of homogeneous Kähler manifolds into Fubini-Study spaces, making use of the representation theory of semi-simple Lie groups.

In $\S 4$, we shall classify completely the Kähler immersions of symmetric Kähler manifolds into Fubini-Study spaces. We state further a theorem of Takagi-Takeuchi [17] on the degrees (For the definition, see $\S 4$ ) of such Kähler immersions. As an application, we reproduce the NakagawaTakagi's classification theorem [15] for Kähler immersions with parallel second fundamental forms.

$\S 5$ is devoted to the study of the nullities $n(f)$, as minimal immersions, of Kähler immersions $f$ of homogeneous Kähler manifolds into FubiniStudy spaces. Let $n_{a}(f)$ be the analytic nullity of $f$ (For the definition, see $\S 5$ ), which was defined by Kimura [8]. We have then an inequality:

$$
n(f) \geqslant n_{a}(f) \geqslant n_{k}(f)
$$

where $n_{k}(f)$ is the Killing nullity of $f$ in the sense of Simons [16]. We shall investigate in detail the relations between these nullities and give certain characterizations of totally geodesic Kähler immersions in terms of these nullities. Furthermore we shall give another proof of a recent result of Kimura to the effect that $n(f)=n_{a}(f)$ and give a formula for $n(f)$. 


\section{$\S 1$. Preliminaries}

In this section, we summarize some known results on Kähler manifolds and Kähler immersions.

Let $(M, g)$ be a Kähler manifold. ${ }^{*}$ An isometric holomorphism of $(M, g)$ onto itself is called an automorphism of $(M, g)$. The group of automorphisms of $(M, g)$ is denoted by Aut $(M, g)$. Let Aut $(M)$ and $\mathrm{I}(M, g)$ denote the group of holomorphisms of the complex manifold $M$ and the one of isometries of the Riemannian manifold $(M, g)$ respectively. The groups Aut $(M, g)$ and $\mathrm{I}(M, g)$ are Lie transformation groups acting on $M$ (cf. Kobayashi [9], Chap. II). If $M$ is compact, Aut (M) is a complex Lie transformation group acting on $M$ and

$$
\operatorname{Aut}^{0}(M, g)=\mathrm{I}^{0}(M, g),
$$

where $\operatorname{Aut}^{\circ}(M, g)$ and $\mathrm{I}^{\circ}(M, g)$ denote the identity components of Aut $(M, g)$ and $\mathrm{I}(M, g)$ respectively (cf. Kobayashi [9], Chap. III). A Kähler manifold $(M, g)$ is called homogeneous if Aut $(M, g)$ acts transitively on $M$. In this case, $g$ is called a homogeneous Kähler metric on $M$. Also a complex manifold $M$ is called homogeneous if Aut $(M)$ acts transitively on $M$.

REMARK 1.1. For a compact complex manifold $M$, the following two assertions are equivalent:

(1) $M$ has a homogeneous Kähler metric,

(2) $M$ is a homogeneous complex manifold and has a Kähler metric, since (1) follows from (2) by a theorem of Borel-Remmert [2].

Let $(M, g)$ be a Kähler manifold. The complex structure tensor of $M$ will be denoted by $J$. Let $a(M)$ be the complex Lie algebra of holomorphic vector fields on $M$. It will be often identified with the real Lie algebra of all smooth vector fields $X$ on $M$ such that the Lie derivative relative to $X$ of $J$ vanishes identically. Let $\mathfrak{a}_{0}(M)$ be the subspace of $\mathfrak{a}(M)$ consisting of all holomorphic vector fields $X$ such that the function $\omega(X)$ vanishes identically for each holomorphic 1-form $\omega$ on $M$. Denote by $g(M, g)$ the Lie algebra of Killing vector fields on $(M, g)$ and put

$$
\mathfrak{g}_{0}(M, g)=\mathfrak{a}_{0}(M) \cap \mathfrak{g}(M, g) .
$$

Let further $c(M, g)$ denote the subspace of $a(M)$ consisting of all holomorphic vector fields which are parallel with respect to the Riemannian connection for $g$. It is a $J$-invariant abelian subalgebra of $a(M)$ contained in $g(M, g)$. If $M$ is compact, $\mathfrak{a}(M)$ and $\mathfrak{g}(M, g)$ are identified with the Lie algebras of

\footnotetext{
*) In this note, a manifold will be always assumed to be connected.
} 
groups Aut $(M)$ and $\operatorname{Aut}^{0}(M, g)=\mathrm{I}^{0}(M, g)$ respectively. Moreover, in this case, $\mathfrak{a}_{0}(M)$ is a $J$-invariant ideal of $\mathfrak{a}(M)$. (cf. Kobayashi [9], Chap. III). With these notations, we have

THEOREM L (Lichnerowicz [11]). Let $(M, g)$ be a compact Kähler manifold of constant scalar curvature. Then, we have direct sums of ideals:

$$
\begin{gathered}
\mathfrak{a}(M)=\mathfrak{c}(M, g) \oplus \mathfrak{a}_{0}(M), \\
\mathfrak{g}(M, g)=\mathfrak{c}(M, g) \oplus \mathfrak{g}_{0}(M, g),
\end{gathered}
$$

and $\mathrm{g}_{0}(M, g)$ is a real form of $\mathfrak{a}_{0}(M)$, i.e.,

$$
\begin{gathered}
\mathfrak{a}_{0}(M)=\mathfrak{g}_{0}(M, g)+J \mathfrak{g}_{0}(M, g), \\
\mathfrak{g}_{0}(M, g) \cap J \mathfrak{g}_{0}(M, g)=\{0\} .
\end{gathered}
$$

We have the following structure theorem on homogeneous Kähler manifolds on which a reductive Lie group acts transitively.

THEOREM M (Matsushima [12]). Let $(M, g)$ be a Kähler manifold and $G$ a connected reductive Lie subgroup of Aut $(M, g)$ acting transitively on $M$. Thus $M$ is identified with the quotient manifold $G / K$, where $K$ is the stabilizer in $G$ of a point of $M$. Then

1) $G$ is the direct product:

$$
G=C \times G_{0}
$$

of the connected center $C$ of $G$ and the largest connected normal semi-simple subgroup $G_{0}$ of $G . \quad K$ is compact connected and coincides with the centralizer in $G_{0}$ of a toral subgroup of $G_{0}$. Thus, $G_{0}$ has no center and $M$ is diffeomorphic with the direct product $C \times M_{0}$ of submanifolds $C$ and $M_{0}=G_{0} / K$. Furthermore $M_{0}$ is simply connected.

2) The complex structure of $M$ induces complex structures on $C$ and $M_{0}$, and the Kähler metric $g$ on $M$ induces a homogeneous flat Kähler metric $h$ on $C$ and a homogeneous Kähler metric $g_{0}$ on $M_{0}$ such that $(M, g)$ is the direct product:

$$
(M, g)=(C, h) \times\left(M_{0}, g_{0}\right)
$$

of Kähler manifolds $(C, h)$ and $\left(M_{0}, g_{0}\right)$.

COROLlary. Let $(M, g)$ be a compact simply connected homogeneous Kähler manifold. Then the identity component $\operatorname{Aut}^{0}(M)$ of $\operatorname{Aut}(M)$ is a connected complex semi-simple Lie group without center and $\operatorname{Aut}^{0}(M, g)$ is a maximal compact subgroup of $\operatorname{Aut}^{0}(M)$. 
Proof. It is known (cf. Kobayashi [9], Chap. III) that

$$
\mathfrak{a}(M)=\mathfrak{a}_{0}(M)
$$

for a compact Kähler manifold $(M, g)$ with the vanishing first Betti number. It follows that for our $(M, g)$ we have $\mathfrak{a}(M)=\mathfrak{a}_{0}(M)$ and hence $\mathfrak{g}(M, g)$ is a real form of $a(M)$, by Theorem $\mathrm{L}$. On the other hand, by Theorem $\mathrm{M}, G=\operatorname{Aut}^{0}(M, g)$ is a compact connected semi-simple Lie group without center. These imply the Corollary.

q.e.d.

REMARK 1.2. Let $(M, g)$ be a compact homogeneous Kähler manifold. Then, by Theorem M, it is the direct product:

$$
(M, g)=(T, h) \times\left(M_{0}, g_{0}\right)
$$

of a flat complex torus $(T, h)$ and a compact simply connected homogeneous Kähler manifold $\left(M_{0}, g_{0}\right)$. Thus we have direct sum decompositions :

(1) $\mathfrak{a}(M)=\mathfrak{a}(T) \oplus \mathfrak{a}\left(M_{0}\right)$,

(2) $g(M, g)=g(T, h) \oplus g\left(M_{0}, g_{0}\right)$,

with $\mathfrak{a}(T)=\mathrm{g}(T, h)$. On the other hand, we have the decomposition of Lichnerowicz :

(1) $\mathfrak{a}(M)=\mathfrak{c}(M, g) \oplus \mathfrak{a}_{0}(M)$,

(2) $g(M, g)=c(M, g) \oplus \mathrm{g}_{0}(M, g)$.

Then, these decompositions coincide in the following sense :

$$
\begin{aligned}
\mathfrak{a}(T) & =\mathfrak{c}(M, g), \\
\mathfrak{a}\left(M_{0}\right) & =\mathfrak{a}_{0}(M), \\
\mathfrak{g}\left(M_{0}, g_{0}\right) & =\mathfrak{g}_{0}(M, g) .
\end{aligned}
$$

Indeed, from the result mentioned in the proof of the above Corollary, we have $\mathfrak{a}\left(M_{0}\right)=\mathfrak{a}_{0}\left(M_{0}\right)$. It follows that $\mathfrak{a}_{0}(M)=\mathfrak{a}\left(M_{0}\right)$, which is semi-simple from the above Corollary. This, together with the decompositions (1) and (1)', implies that both $\mathfrak{a}(T)$ and $\mathfrak{c}(M, g)$ are the center of $\mathfrak{a}(M)$. Thus $\mathfrak{a}(T)=$ $\mathfrak{c}(M, g)$, and hence $\operatorname{dim} g\left(M_{0}, g_{0}\right)=\operatorname{dim} \mathrm{g}_{0}(M, g)$. Since $\mathfrak{g}\left(M_{0}, g_{0}\right) \subset \mathfrak{a}\left(M_{0}\right) \cap$ $\mathfrak{g}(M, g)=\mathfrak{a}_{0}(M) \cap \mathfrak{g}(M, g)=\mathfrak{g}_{0}(M, g)$, we get $\mathfrak{g}\left(M_{0}, g_{0}\right)=\mathfrak{g}_{0}(M, g)$.

Let $(M, g)$ and $\left(M^{\prime}, g^{\prime}\right)$ be Kähler manifolds. An isometric holomorphism between $(M, g)$ and $\left(M^{\prime}, g^{\prime}\right)$ is called an equivalence. $(M, g)$ and $\left(M^{\prime}, g^{\prime}\right)$ are said to be equivalent, denoted by $(M, g) \cong\left(M^{\prime}, g^{\prime}\right)$, if there exists an equivalence between them. An equivalence class of Kähler manifolds is called a Kähler structure. A Kähler structure of homogeneous Kähler manifolds is called a homogeneous Kähler structure.

Let $P_{N}(C)$ be the $N$-dimensional complex projective space and $g_{c}$ the Fubini-Study metric on $P_{N}(C)$ of constant holomorphic sectional curvature 
$c>0$. The Kählar manifold $\left(P_{N}(C), g_{c}\right)$ will be abbreviated by $P_{N}(c)$ and called a Fubini-Study space. The groups Aut $\left(P_{N}(C)\right)=\operatorname{Aut}^{0}\left(P_{N}(C)\right)$ and $\operatorname{Aut}\left(P_{N}(c)\right)=\operatorname{Aut}^{0}\left(P_{N}(c)\right)$ are identified in a natural way with

$$
\mathrm{PL}(N+1, C)=\mathrm{SL}(N+1, C) /\left\{\varepsilon 1_{N+1} ; \varepsilon^{N+1}=1\right\}
$$

and

$$
\mathrm{PU}(N+1)=\mathrm{SU}(N+1) /\left\{\varepsilon 1_{N+1} ; \varepsilon^{N+1}=1\right\}
$$

respectively.

Take $c>0$ and fix it once for all. In this note we are mainly interested in a Kähler immersion, i.e., an isometric holomorphic immersion, of a Kähler manifold into a Fubini-Study space $P_{N}(c)$. Two Kähler immersions $f:(M, g) \rightarrow P_{N}(c)$ and $f^{\prime}:\left(M^{\prime}, g^{\prime}\right) \rightarrow P_{N^{\prime}}(c)$ are said to be equivalent, denoted by $f \cong f^{\prime}$, if $N=N^{\prime}$ and if there exist an equivalence $\varphi:(M, g) \rightarrow\left(M^{\prime}, g^{\prime}\right)$ and $\Phi \in \operatorname{Aut}\left(P_{N}(c)\right)$ such that $\Phi \circ f=f^{\prime} \circ \varphi$.

Let $M$ be a complex manifold and $f$ a holomorphic immersion of $M$ into $P_{N}(C)$. The dimension of the smallest linear submanifold of $P_{N}(C)$ containing $f(M)$ will be denoted by $N(f) . \quad f$ is said to be $f u l l$ if $N(f)=N$.

REMARK 1.3. Let $f: M \rightarrow P_{N}(C)$ be a full holomorphic immersion of a complex manifold $M$. Then

(a) For any open subset $U$ of $M$, the restriction of $f$ on $U$ is also a full immersion of $U$ into $P_{N}(C)$.

(b) For $x \in \operatorname{Aut}(M)$, there exists at most one $\Phi \in$ Aut $\left(P_{N}(C)\right)$ such that $f \circ x=\Phi \circ f$.

(a) follows from the analyticity of $f$. For the proof of (b), it suffices to show that if $\Phi \in$ Aut $\left(P_{N}(C)\right)$ fixes each point of $f(M)$ then $\Phi=e$. This can be proved by the same method as in Nakagawa-Takagi [15], Theorem 4.3 .

The following theorem plays a fundamental role in the study of Kähler submanifolds of $P_{N}(c)$.

Theorem C (Calabi [4]). (I) (Rigidity theorem). Let $f:(M, g) \rightarrow$ $P_{N}(c)$ and $f^{\prime}:(M, g) \rightarrow P_{N^{\prime}}(c)$ be two full Kähler immersions of the same Kähler manifold $(M, g)$. Then, $N=N^{\prime}$ and there exists a unique $\Phi \in$ Aut $\left(P_{N}(c)\right)$ such that $\Phi \circ f=f^{\prime}$.

(II) (Extension theorem). Let $(M, g)$ be a simply conected Kähler manifold with real analytic Kähler metric $g$, and $U$ a connected open subset of $M$. Then each local Kähler immersion: $(U, g) \rightarrow P_{N}(c)$ is extended uniquely to a global Kähler immersion: $(M, g) \rightarrow P_{N}(c)$. 
For a Kähler manifold $(M, g)$ which is Kähler immersible into a Fubini-Study space, the dimension of the target Fubini-Study space of a full Kähler immersion of it depends only on the Kähler structure of $(M, g)$, in view of the Theorem (I). This dimension will be denoted by $N(M, g)$. The following corollaries are the immediate consequences of the Theorem (I) and Remark $1.3(b)$.

COROLlaRY 1. Let $f:(M, g) \rightarrow P_{N}(c)$ be a Kähler immersion of a Kähler manifold $(M, g)$. Then

$$
N(f)=N(M, g)
$$

Corollary 2. Let $f:(M, g) \rightarrow P_{N}(c)$ and $f^{\prime}:\left(M^{\prime}, g^{\prime}\right) \rightarrow P_{N^{\prime}}(c)$ be two full Kähler immersions. Then $f \cong f^{\prime}$ if and only if $(M, g) \cong\left(M^{\prime}, g^{\prime}\right)$.

Corollary 3. Let $f:(M, g) \rightarrow P_{N}(c)$ be a full Kähler immersion of a Kähler manifold $(M, g)$. Then there exists a unique Lie group homomorphism $\rho: \operatorname{Aut}^{0}(M, g) \rightarrow \operatorname{Aut}\left(P_{N}(c)\right)$ with discrete kernel such that

$$
\rho(x) \circ f=f \circ x \quad \text { for each } x \in \operatorname{Aut}^{0}(M, g),
$$

i.e., $f$ is $\operatorname{Aut}^{0}(M, g)$-equivariant relative to $\rho$.

Note that the discreteness of Kernel $\rho$ follows from the relation (*) and from that $f$ is locally an imbedding.

Let $f:(M, g) \rightarrow P_{N}(c)$ be a Kähler immersion. Then the pull back $f^{*} T P_{N}(C)$ of the tangent bundle $T P_{N}(C)$ of $P_{N}(C)$ is identified, as a smooth vector bundle, with the Whitney sum:

$$
f^{*} T P_{N}(C)=T M \oplus N M
$$

of the tangent bundle $T M$ of $M$ and the normal bundle $N M$ of $f$. Let $\Gamma\left(f^{*} T P_{N}(C)\right)$ denote the space of holomorphic sections of $f^{*} T P_{N}(C)$ and $f^{*}: \mathfrak{a}\left(P_{N}(C)\right) \rightarrow \Gamma\left(f^{*} T P_{N}(C)\right)$ the pull back map. Note that $f^{*}$ is a $C$-linear map. Let $a\left(P_{N}(C), M\right)$ denote the subalgebra of $a\left(P_{N}(C)\right)$ consisting of those vector fields $X \in \mathfrak{a}\left(P_{N}(C)\right)$ such that $f^{*} X$ is tangent to $M$. In the same way, $\mathrm{g}\left(P_{N}(c), M\right)$ denotes the subalgebra of $\mathrm{g}\left(P_{N}(c)\right)$ consisting of those $X \in \mathfrak{g}\left(P_{N}(c)\right)$ such that the pull back $f^{*} X$ is tangent to $M$. Then the restrictions define the homomorphisms $\sigma: \mathfrak{a}\left(P_{N}(C), M\right) \rightarrow \mathfrak{a}(M)$ and $\sigma: \mathfrak{g}\left(P_{N}(c), M\right) \rightarrow$ $\mathrm{g}(M, g)$. Note that if $M$ is compact, the latter map is surjective in virtue of the above Corollary $3 . \quad f$ is said to be $a(M)$-equivariant if there exists a complex homomorphism $\rho: \mathfrak{a}(M) \rightarrow \mathfrak{a}\left(P_{N}(C), M\right)$ such that

$$
\sigma(\rho(X))=X \quad \text { for each } X \in \mathfrak{a}(M) .
$$


Note that such $\rho$ is always injective.

\section{§ 2. Homogeneous Kähler submanifolds of $\boldsymbol{P}_{N}(\boldsymbol{c})$}

In this section we shall find all homogeneous Kähler submanifolds of Fubini-Study spaces. We begin with some lemmas.

LEMMA 2.1. Let $f:(M, g) \rightarrow P_{N}(c)$ be a Kähler immersion of a compact Kähler manifold $(M, g)$ of constant scalar curvature. Then

1) $c(M, g)=\{0\}$.

2) $\operatorname{Aut}^{0}(M)$ is a connected complex reductive Lie group with a maximal compact subgroup $\operatorname{Aut}^{0}(M, g)=\mathrm{I}^{0}(M, g)$.

3) If $f$ is a full immersion, then there exists a unique complex Lie group homomorphism $\rho: \operatorname{Aut}^{0}(M) \rightarrow \operatorname{Aut}\left(P_{N}(C)\right)$ with discrete kernel such that $\rho\left(\operatorname{Aut}^{0}(M, g)\right) \subset \operatorname{Aut}\left(P_{N}(c)\right)$ and that

$$
\rho(x) \circ f=f \circ x \quad \text { for each } x \in \operatorname{Aut}^{0}(M),
$$

i.e., $f$ is $\operatorname{Aut}^{0}(M)$-equivariant relative to $\rho$. Thus $f$ is $\mathfrak{a}(M)$-equivariant.

4) If $f$ is a full imbedding, then the above homomorphism $\rho$ is injective.

Proof. We may assume that $f$ is full. Then, by Remark 1.3, the homomorphism $\sigma: \mathfrak{a}\left(P_{N}(C), M\right) \rightarrow \mathfrak{a}(M)$ is $C$-linear injective. The differential of the homomorphism $\rho$ in Corollary 3 of Theorem $\mathrm{C}$ will be denoted by the same $\rho: g(M, g) \rightarrow \mathfrak{g}\left(P_{N}(C)\right)$. It is injective and satisfies $\sigma \rho(X)=X$ for each $X \in g(M, g)$. Therefore, for $X \in \mathfrak{c}(M, g)$, we have

$$
\sigma J \rho X=J \sigma \rho X=J X=\sigma \rho J X,
$$

and hence $J \rho X=\rho J X$. Thus $\rho(c(M, g))$ is a $J$-invariant subalgebra of $\mathfrak{a}\left(P_{N}(C)\right)=\mathfrak{g l}(N+1, C)$ contained in $g\left(P_{N}(c)\right)=\mathfrak{g} \mathfrak{u}(N+1)$. Therefore it must be $\{0\}$, and hence $c(M, g)=\{0\}$. This, together with Theorem L, implies 2). Since 4) follows from the property of $\rho$, it remains to prove 3). Let $\tilde{\tau}$ : $\mathrm{SL}(N+1, C) \rightarrow \mathrm{SL}\left(\left(\begin{array}{c}2 N+1 \\ N+1\end{array}\right), C\right)$ be the $(N+1)$-th symmetric power of the identity representation of $\operatorname{SL}(N+1, C)$. It induces a faithful representation $\tau: \operatorname{Aut}\left(P_{N}(C)\right) \rightarrow \operatorname{SL}\left(\left(\begin{array}{c}2 N+1 \\ N+1\end{array}\right), C\right)$ with $\tau\left(\operatorname{Aut}\left(P_{N}(c)\right)\right) \subset \mathrm{SU}\left(\left(\begin{array}{c}2 N+1 \\ N+1\end{array}\right)\right)$. The representation $\tau \circ \rho: \operatorname{Aut}^{0}(M, g) \rightarrow \mathrm{SL}\left(\left(\begin{array}{c}2 N+1 \\ N+1\end{array}\right), C\right)$ of $\operatorname{Aut}^{0}(M, g)$ is uniquely extended to a holomorphic representation $P: \operatorname{Aut}^{0}(M) \rightarrow$ $\mathrm{SL}\left(\left(\begin{array}{c}2 N+1 \\ N+1\end{array}\right), C\right)$ with $P\left(\operatorname{Aut}^{0}(M)\right) \subset \tau\left(\operatorname{Aut}\left(P_{N}(C)\right)\right)$, in virtue of 2$)$. Now $P$ 
induces in a natural way a complex Lie group homomorphism $\rho: \operatorname{Aut}^{0}(M) \rightarrow$ Aut $\left(P_{N}(C)\right)$, which is the required one.

q.e.d.

Lemma 2.2 (cf. Calabi [4]). Any flat Kähler manifold does not admit a Kähler immersion into a Fubini-Study space.

Proof. Suppose that a flat Kähler manifold $(M, g)$ would admit a Kähler immersion $f:(M, g) \rightarrow P_{N}(c)$. By Theorem $\mathrm{C}$ (II), we may assume that $(M, g)$ is a flat complex affine space. Then $c(M, g)$ is the Lie algebra of the subgroup of $\operatorname{Aut}^{0}(M, g)$ of translations of $M$, and hence $\operatorname{dim}_{C} \mathfrak{c}(M, g)$ $=\operatorname{dim}_{C} M$. We may further assume that $f$ is full. Now we would conclude $\mathfrak{c}(M, g)=\{0\}$ in the same way as the proof of the assertion 1$)$ of Lemma 2.1. This is a contradiction. q.e.d.

LEMMA 2.3. Let $f:(M, g) \rightarrow P_{N}(c)$ be a Kähler immersion of a homogeneous Kähler manifold $(M, g)$. Then

1) $M$ is compact and simply connected.

2) $f$ is an imbedding.

Proof. We may assume that $f$ is full. By Corollary 3 of Theorem $\mathrm{C}$, we have a Lie group homomorphism $\rho: \operatorname{Aut}^{0}(M, g) \rightarrow \operatorname{Aut}\left(P_{N}(c)\right)$ with discrete kernel such that

$$
\rho(x) \circ f=f \circ x \quad \text { for each } x \in \operatorname{Aut}^{0}(M, g) .
$$

1) Taking the differential of $\rho$, we get an injective homomorphism from the Lie algebra $g$ of $\operatorname{Aut}^{0}(M, g)$ into $\mathfrak{H} \mathfrak{H}(N+1)$. Hence $\mathfrak{g}$ is reductive and decomposed to the direct sum :

$$
\mathfrak{g}=\mathfrak{c} \oplus \mathfrak{g}_{0}
$$

of the center $c$ and the compact semi-simple ideal $g_{0}$. It follows from Theorem $M$ that $(M, g)$ is decomposed to the direct product:

$$
(M, g)=(C, h) \times\left(M_{0}, g_{0}\right)
$$

of a flat Kähler manifold $(C, h)$ and a compact simply connected homogeneous Kähler manifold $\left(M_{0}, g_{0}\right)$. Taking a point $p_{0} \in M_{0}$, we define a holomorphic $\operatorname{map} f_{C}: C \rightarrow P_{N}(C)$ by

$$
f_{C}(c)=f\left(c \times p_{0}\right) \quad \text { for } c \in C .
$$

This defines a Kähler immersion $f_{C}:(C, h) \rightarrow P_{N}(c)$. It follows from Lemma 2.2 that $C=\{e\}$ and hence $M=M_{0}$ is compact and simply connected.

2) Let $G=\operatorname{Aut}^{0}(M, g)$. Take a point $o \in M$, and put 


$$
K=\{x \in G ; x o=o\},
$$

and

$$
K^{\prime}=\{x \in G ; \rho(x) f(o)=f(o)\} .
$$

Then $K$ is a subgroup of $K^{\prime}$ and the image $M^{\prime}=f(M)$ is identified with the quotient manifold $G / K^{\prime}$. The map $f: M \rightarrow M^{\prime}$ is nothing but the natural covering map: $G / K \rightarrow G / K^{\prime}$. Hence $M^{\prime}$ is a complex submanifold of $P_{N}(C)$ and it has a Kähler metric $g^{\prime}$ induced from the one on $M$. Applying the above 1) to the Kähler imbedding $f^{\prime}:\left(M^{\prime}, g^{\prime}\right) \longrightarrow P_{N}(c)$, we know that $M^{\prime}$ is simply connected. Thus the covering map $f: M \rightarrow M^{\prime}$ is trivial, and hence $f$ is an imbedding.

q.e.d.

Summarizing these lemmas, we have the following

THEOREM 2.1. Let $f:(M, g) \rightarrow P_{N}(c)$ be a Kähler immersion of a homogeneous Kähler manifold $(M, g)$. Then

1) $M$ is compact and simply connected.

2) $\operatorname{Aut}^{0}(M)$ is a connected complex semi-simple Lie group without center. Aut $^{0}(M, g)$ is a connected compact semi-simple Lie group without center, which is a maximal compact subgroup of $\operatorname{Aut}^{0}(M)$.

3) $f$ is an imbedding.

4) If $f$ is full, then there exists a unique injective complex Lie group homomorphism $\rho: \operatorname{Aut}^{0}(M) \rightarrow \operatorname{Aut}\left(P_{N}(C)\right)$ with $\rho\left(\operatorname{Aut}^{0}(M, g)\right) \subset \operatorname{Aut}\left(P_{N}(c)\right)$ such that

$$
\rho(x) \circ f=f \circ x \quad \text { for each } x \in \operatorname{Aut}^{0}(M),
$$

i.e., $f$ is $\operatorname{Aut}^{0}(M)$-equivariant relative to $\rho$.

COROLlaRY. If a complete, locally homogeneous Kähler manifold $(M, g)$ admits a Kähler immersion $f:(M, g) \rightarrow P_{N}(c)$, then it is a homogeneous Kähler manifold.

Proof. Let $\pi:(\tilde{M}, \tilde{g}) \rightarrow(M, g)$ be the universal Kähler covering of $(M, g)$. From the assumption, $(\tilde{M}, \tilde{g})$ is a homogeneous Kähler manifold. Applying the Theorem to the Kähler immersion $\tilde{f}=f \circ \pi:(M, g) \rightarrow P_{N}(c)$, we know that $\tilde{f}$ is an imbedding. Therefore the covering $\pi$ is trivial and hence $M=\tilde{M}$ is a homogeneous Kähler manifold.

q.e.d.

REMARK 2.1. Let $f:(M, g) \rightarrow P_{N}(c)$ be a totally geodesic Kähler immersion of a complete Kähler manifold $(M, g)$ with $\operatorname{dim}_{C} M=n$. Then, $(M, g)$ $=P_{n}(c)$ and $f$ is an imbedding. Indeed, applying the same argument as the above for our $f$, we know that $(\tilde{M}, \tilde{g})=P_{n}(c)$. Hence, by Theorem 2.1, 
$M=\tilde{M}$ and $f$ is an imbedding.

Next we recall the standard construction of Kähler imbeddings of compact simply connected homogeneous Kähler manifolds (cf. BorelHirzebruch [1], Takeuchi [18], Nakagawa-Takagi [15]).

Let $\Pi$ be a Dynkin diagram and $\Pi_{0}$ a subdiagram of $\Pi$. The pair $\left(\Pi, \Pi_{0}\right)$ is said to be effective if $\Pi_{0}$ does not contain any irreducible component of $\Pi$. Consider an effective pair $\left(\Pi, \Pi_{0}\right)$ of Dynkin diagrams and a map $p: \Pi-\Pi_{0} \rightarrow \boldsymbol{Z}^{+}$, where $\boldsymbol{Z}^{+}$denotes the set of positive integers. Such a triple $\left(\Pi, \Pi_{0} ; p\right)$ is called an admissible triple. In the following, for an admissible triple $\left(\Pi, \Pi_{0} ; p\right)$, we construct a full Kähler imbedding of a compact simply connected homogeneous Kähler manifold into a Fubini-Study space.

Let $\Sigma$ be the root system with the fundamental root system $\Pi$. Choose a lexicographic order $>$ on $\Sigma$ such that the set of simple roots with respect to $>$ coincides with $\Pi$. Take a compact semi-simple Lie algebra $g$ with the root system $\Sigma$. Let $t$ be a maximal abelian subalgebra of $g$ and denote by $\mathrm{g}^{c}$ and $t^{c}$ the complexifications of $\mathrm{g}$ and $t$ respectively. We identify a weight of $\mathrm{g}^{c}$ relative to the Cartan subalgebra $t^{c}$ with an element of $\sqrt{-1} t$ by means of the duality defined by the Killing form (, ) of $g^{c}$. Thus the root system of $\mathrm{g}^{c}$ relative to $t^{C}$ is a subset of $\sqrt{-1}$ t and identified with $\Sigma$. Let $\left\{\Lambda_{\alpha}\right\}_{\alpha \in \Pi}$ $\subset \sqrt{-1} t$ be the fundamental weights of $g^{c}$ corresponding to $\Pi$ :

$$
\frac{2\left(\Lambda_{\alpha}, \beta\right)}{(\beta, \beta)}= \begin{cases}1 & \text { if } \alpha=\beta, \\ 0 & \text { if } \alpha \neq \beta .\end{cases}
$$

Put

$$
\Sigma^{+}=\{\alpha \in \Sigma ; \alpha>0\}
$$

and

$$
\Sigma_{0}=\Sigma \cap\left\{\Pi_{0}\right\}_{Z},
$$

where $\left\{\Pi_{0}\right\}_{Z}$ denotes the subgroup of $\sqrt{-1}$ t generated by $\Pi_{0}$. Define a subalgebra $\mathfrak{u}$ of $\mathrm{g}^{c}$ by

$$
\mathfrak{u}=t^{C}+\sum_{\alpha \in \Sigma_{0} \cup \Sigma^{+}} g_{\alpha}^{C},
$$

where $g_{\alpha}^{c}$ denotes the root space of $g^{c}$ for $\alpha \in \Sigma$. Let $G^{c}$ be the connected complex semi-simple Lie group without center, whose Lie algebra is $\mathrm{g}^{c}$, and $U$ the connected (closed) complex subgroup of $G^{c}$ generated by $\mathfrak{u}$. We define a complex manifold $M$ by 


$$
M=G^{c} / U
$$

which is known to be compact and simply connected. $G^{c}$ acts effectively on $M$, since $G^{c}$ has no center. The origin $U$ of $M$ will be denoted by $o$. Let $G$ be a compact connected semi-simple subgroup of $G^{C}$ generated by $\mathfrak{g}$ and put $K=G \cap U$. Then $K$ is connected and $G$ acts on $M$ transitively, and hence the natural map : $G / K \rightarrow G^{c} / U$ induces an identification: $M=G / K$ as a smooth manifold. The complexification $\mathfrak{f}^{C}$ of the Lie algebra $\mathfrak{1}$ of $K$ is given by

$$
\mathfrak{l}=\mathfrak{t}^{C}+\sum_{\alpha \in \Sigma_{0}} \mathfrak{g}_{\alpha}^{C} .
$$

Note that the construction of $M$ depends only on the effective pair $\left(\Pi, \Pi_{0}\right)$ and not on the map $p$. This homogeneous complex manifold $M$ will be said to be associated to $\left(\Pi, \Pi_{0}\right)$.

Now, making use of the map $p: \alpha \mapsto p_{\alpha}\left(\alpha \in \Pi-\Pi_{0}\right)$, we define an element $\Lambda \in \sqrt{-1}$ t by

$$
\Lambda=\sum_{\alpha \in \Pi-\Pi_{0}} p_{\alpha} \Lambda_{\alpha}
$$

Let $\rho: \mathfrak{g} \rightarrow \mathfrak{g} \mathfrak{H}(N+1)$ be an irreducible unitary representation of $\mathfrak{g}$ such that its $C$-linear extension $\rho: \mathfrak{g}^{c} \rightarrow \mathfrak{g} \backslash(N+1, C)$ has the highest weight $\Lambda$. Let $\tilde{G}^{c}$ be the universal covering group of $G^{c}$ and $\tilde{G}$ the (simply) connected subgroup of $\tilde{G}^{c}$ generated by $g$. Extend $\rho$ to a holomorphic representation $\tilde{\rho}: G^{c} \rightarrow \mathrm{SL}(N+1, C)$. By virtue of the irreducibility, it induces a complex Lie group homomorphism $\rho: G^{c} \rightarrow \mathrm{PL}(N+1, C)$ such that the diagram:

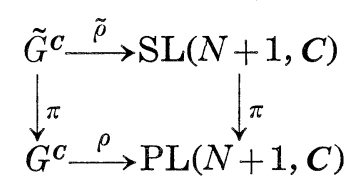

is commutative, where the $\pi$ 's denote the covering homomorphisms. This induces then a commutative diagram:

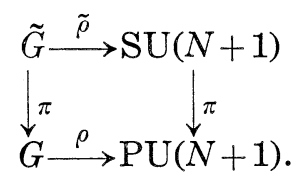

Let $P_{N}(C)=C^{N^{+1}}-\{0\} / C^{*}$, where $C^{*}$ denotes the group of non-zero complex numbers, be the complex projective space associated to the representation space $C^{N+1}$ of $\tilde{\rho}$. For a vector $v \in C^{N+1}-\{0\}$, the equivalence class of $v$ will 
be denoted by $[v] \in P_{N}(C)$. Taking a highest weight vector $v_{\Lambda} \in C^{N+1}-\{0\}$, we can define a holomorphic imbedding $f: M \rightarrow P_{N}(C)$ by

$$
f(x o)=\rho(x)\left[v_{A}\right] \quad \text { for } x \in G^{c} .
$$

It is $G^{c}$-equivariant relative to $\rho$ :

$$
f \circ x=\rho(x) \circ f \quad \text { for each } x \in G^{c} .
$$

Let us introduce a Kähler metric $g$ on $M$ in such a way that the map $f:(M, g) \rightarrow P_{N}(c)$ becomes a Kähler imbedding. Then, since $\rho(G) \subset$ $P U(N+1)=$ Aut $\left(P_{N}(c)\right)$, we have $G \subset \operatorname{Aut}^{0}(M, g)$. Thus $(M, g)$ is a homogeneous Kähler manifold. The irreducibility of $\tilde{\rho}$ implies the fullness of $f$. The Kähler imbedding $f$ is said to be associated to $\left(\Pi, \Pi_{0} ; p\right)$.

Let $\left(\Pi, \Pi_{0} ; p\right)$ and $\left(\Pi^{\prime}, \Pi_{0}^{\prime} ; p^{\prime}\right)$ be admissible triples. They are said to be equivalent, denoted by $\left(\Pi, \Pi_{0} ; p\right) \cong\left(\Pi^{\prime}, \Pi_{0}^{\prime} ; p^{\prime}\right)$, if there exists an equivalence $\psi: \Pi \rightarrow \Pi^{\prime}$ of Dynkin diagrams such that $\psi \Pi_{0}=\Pi_{0}^{\prime}$ and $p=p^{\prime} \circ \psi$ on $\Pi-\Pi_{0}$.

With these definitions, we have

THEOREM 2.2. The correspondence described above gives a surjection from the set of equivalence classes of admissible triples to the set of equivalence classes of full Kähler immersions of homogeneous Kähler manifolds into Fubini-Study spaces (of constant holomorphic sectional curvatures c).

Proof. (i) Let $\left(\Pi, \Pi_{0} ; p\right)$ and $\left(\Pi^{\prime}, \Pi_{0}^{\prime} ; p^{\prime}\right)$ be admissible triples. The objects for $\left(\Pi^{\prime}, \Pi_{0}^{\prime} ; p^{\prime}\right)$ will be denoted by the same symbols as those for $\left(\Pi, \Pi_{0} ; p\right)$ but with primes. We shall show that $\left(\Pi, \Pi_{0} ; p\right) \cong\left(\Pi^{\prime}, \Pi_{0}^{\prime} ; p^{\prime}\right) \mathrm{im}$ plies $f \cong f^{\prime}$.

From the assumption, there exists an equivalence $\psi: \Pi \rightarrow \Pi^{\prime}$ with $\psi \Pi_{0}$ $=\Pi_{0}^{\prime}, p^{\prime} \circ \psi=p$. Then there exists an isomorphism from $\mathrm{g}^{C}$ onto $\mathrm{g}^{\prime C}$, denoted by the same $\psi$, such that $\psi \mathrm{g}=\mathfrak{g}^{\prime}, \psi t=\mathfrak{t}^{\prime}, \psi \Sigma=\Sigma^{\prime}, \psi \Pi=\Pi^{\prime}, \psi \Pi_{0}=\Pi_{0}^{\prime}$, $\psi \Lambda=\Lambda^{\prime}$ and that $\psi \mid \Pi$ coincides with the original equivalence $\psi: \Pi \rightarrow \Pi^{\prime}$. Thus we have also $\psi \mathfrak{H}=\mathfrak{H}^{\prime}$. The extension $\tilde{\psi}: \tilde{G}^{c} \rightarrow \tilde{G}^{\prime c}$ of the isomorphism $\psi: \mathfrak{g}^{C} \rightarrow \mathrm{g}^{\prime C}$ induces an isomorphism $\psi: G^{C} \rightarrow G^{\prime c}$ such that the diagram:

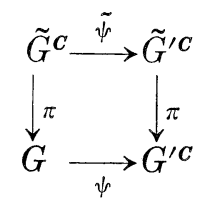

is commutative. It satisfies $\psi G=G^{\prime}$ and $\psi U=U^{\prime}$. It follows from $\psi \Lambda=\Lambda^{\prime}$ that irreducible representations $\rho^{\prime} \circ \psi$ and $\rho$ of $\mathrm{g}^{c}$ have the same highest 
weights relative to $t^{C}$. Therefore, $N=N^{\prime}$ and there exists $\tilde{\Phi} \in \mathrm{SU}(N+1)$ such that

$$
\rho^{\prime}(\psi(X))=\tilde{\Phi} \rho(X) \tilde{\Phi}^{-1} \quad \text { for } X \in \mathfrak{g}^{c} .
$$

Putting $\Phi=\pi(\tilde{\Phi}) \in \mathrm{PU}(N+1)$, we get $\left[v_{\Lambda^{\prime}}^{\prime}\right]=\Phi\left[v_{\Lambda}\right]$. We define a holomorphism $\varphi: M \rightarrow M^{\prime}$ by

$$
\varphi(x o)=\psi(x) o^{\prime} \quad \text { for } x \in G^{c} .
$$

We have then a commutative diagram :

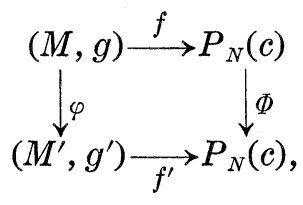

and hence $\varphi:(M, g) \rightarrow\left(M^{\prime}, g^{\prime}\right)$ is an equivalence of Kähler manifolds. Thus we conclude $f \cong f^{\prime}$.

(ii) Let $f:(M, g) \rightarrow P_{N}(c)$ be a full Kähler immersion of a homogeneous Kähler manifold $(M, g)$. We shall associate to $f$ an admissible triple $\left(\Pi, \Pi_{0} ; p\right)$ in a canonical way.

THEOREM 2.1 implies: $M$ is compact and simply connected; $f$ is an imbedding; Put $G^{c}=\operatorname{Aut}^{0}(M)$ and $G=\operatorname{Aut}^{0}(M, g)$, then $G^{c}$ is a connected complex semi-simple Lie group without center and $G$ is a maximal compact subgroup of $G^{c}$; Thus the Lie algebra $\mathrm{g}^{c}$ of $G^{c}$ is the complexification of the Lie algebra $g$ of $G$; There exists a unique injective complex Lie group homomorphism $\rho: G^{c} \rightarrow \mathrm{PL}(N+1, C)$ with $\rho(G) \subset \mathrm{PU}(N+1)$ such that $\rho(x) \circ f$ $=f \circ x$ for each $x \in G^{c}$. Take a point $o \in M$ and put

$$
U=\left\{x \in G^{c} ; x o=o\right\},
$$

and $K=G \cap U$. Let $\mathfrak{u}$ and $\mathfrak{f}$ be the Lie algebras of $U$ and $K$ respectivity. By Theorem M, $K$ is the centralizer of a toral subgroup of $G$, and hence contains a maximal abelian subalgebra $\downarrow$ of $\mathfrak{g}$. Let $\Sigma$ be the root system of $\mathrm{g}^{c}$ relative to the Cartan subalgebra $\rfloor^{c}$, which will be identified with a subset of $\sqrt{-1}$ t by means of the Killing form (, ) of $\mathrm{g}^{c}$. Then it is known (cf. Borel-Hirzebruch [1]) that there exists a fundamental root system $\Pi$ for $\Sigma$ and a subsystem $\Pi_{0} \subset \Pi$ such that

$$
\mathfrak{U}=\mathfrak{t}^{C}+\sum_{\alpha \in \Sigma_{0} \cup \Sigma+} \mathfrak{g}_{\alpha}^{C},
$$

where $\Sigma^{+}$and $\Sigma_{0}$ are defined from $\left(\Pi, \Pi_{0}\right)$ in the same way as before. In 
this case, the pair $\left(\Pi, \Pi_{0}\right)$ is effective and the complexification $\mathfrak{f}^{c}$ of $\mathfrak{H}^{\mathrm{i}}$ is given by

$$
\mathfrak{l}^{C}=\mathfrak{t}^{C}+\sum_{\alpha \in \Sigma_{0}} \mathfrak{g}_{\alpha}^{C} .
$$

Now let us consider the differential of $\rho$, and denote it also by $\rho: \mathrm{g}^{c} \rightarrow$ grl $(N+1, C)$. Let

$$
f(o)=[v], \quad v \in C^{N+1}-\{0\} .
$$

Then $\mathfrak{u}$ is characterized by

$$
\mathfrak{U}=\left\{X \in \mathfrak{g}^{c} ; \rho(X) v \in C v\right\} .
$$

We prove first that $\rho$ is an irreducible representation of $\mathrm{g}^{c}$. Decompose the representation space $C^{N+1}$ of $\rho$ into the direct sum :

$$
C^{N+1}=\sum_{i=1}^{m} V_{i}
$$

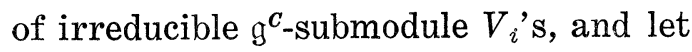

$$
v=\sum_{i=1}^{m} v_{i}, \quad v_{i} \in V_{i}(1 \leqslant i \leqslant m)
$$

Then each $v_{i}$ does not vanish in virtue of the fullness of $f$, and hence, for each $i, v_{i}$ is a weight vector belonging to a weight, say $\lambda_{i}$, of $\mathrm{g}^{C}$-module $V_{i}$. But since $g_{\alpha}^{c} \subset \mathfrak{u}$ for each $\alpha \in \Sigma^{+}, \lambda_{i}$ is the highest weight of $V_{i}$ for each $i$. The characterization (2.2) implies also $\lambda_{1}=\cdots=\lambda_{m}$, and hence the $\mathrm{g}^{C}$-module $V_{i}$ 's are equivalent one another. It follows that the vectors of the form $\rho\left(X_{1}\right) \cdots \rho\left(X_{k}\right) v, X_{i} \in \mathrm{g}^{c}$, span over $C$ a subspace $V^{\prime}$ of $C^{N+1}$ with $\operatorname{dim} V^{\prime}=$ $\operatorname{dim} V_{1}$. Now the fullness of $f$ implies $m=1$, and hence $\rho$ is irreducible. Incidentally we have proved

$$
f(o)=\left[v_{A}\right]
$$

where $v_{A} \in C^{N+1}-\{0\}$ is a weight vector belonging to the highest weight $\Lambda$ of $\rho$. Now it is easy to show from (2.1) and (2.2) that $\Lambda$ is of the form:

$$
\Lambda=\sum_{\alpha \in \Pi-\Pi_{0}} p_{\alpha} \Lambda_{\alpha}, \quad p_{\alpha} \in Z^{+},
$$

where the $\Lambda_{\alpha}$ 's are the fundamental weights corresponding to $\Pi$. Defining a map $p: \Pi-\Pi_{0} \rightarrow Z^{+}$by $\alpha \mapsto p_{\alpha}$, we get an admissible triple $\left(\Pi, \Pi_{0} ; p\right)$. Note that there exists a holomorphic representation $\tilde{\rho}: \tilde{G}^{c \rightarrow} \mathrm{SL}(N+1, C)$ of the universal covering group $\tilde{G}^{c}$ of $G^{c}$ such that the diagram: 


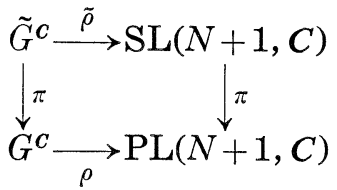

is commutative. This induces a commutative diagram :

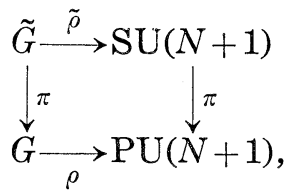

where $\tilde{G}$ is the (simply) connected subgroup of $\tilde{G}^{c}$ generated by g.

(iii) It is easy to see that if $\left(\Pi, \Pi_{0} ; p\right)$ is associated to $f$, and if $f^{\prime}$ is associated to $\left(\Pi, \Pi_{0} ; p\right)$, then $f \cong f^{\prime}$. This proves the Theorem. q.e.d.

We prove the following lemma for a later use.

LEMMA 2.4. Let $f:(M, g) \rightarrow P_{N}(c)$ and $f^{\prime}:\left(M^{\prime}, g^{\prime}\right) \rightarrow P_{N^{\prime}}(c)$ be full Kähler immersions of homogeneous Kähler manifolds, and let $\left(\Pi, \Pi_{0} ; p\right)$ and $\left(\Pi^{\prime}, \Pi_{0}^{\prime} ; p^{\prime}\right)$ be admissible triples associated to $f$ and $f^{\prime}$ respectively. Then $f \cong f^{\prime}$ implies $\left(\Pi, \Pi_{0} ; p\right) \cong\left(\Pi^{\prime}, \Pi_{0}^{\prime}, p^{\prime}\right)$.

Proof. The objects in the construction (ii) in Theorem 2.2 for $f^{\prime}$ will be denoted by the same symbols as those for $f$ but with primes. It follows from the assumption that $N=N^{\prime}$ and there exist an equivalence $\varphi:(M, g) \rightarrow$ $\left(M^{\prime}, g^{\prime}\right)$ and $\Phi \in \mathrm{PU}(N+1)$ such that $\Phi \circ f=f^{\prime} \circ \varphi$. We define a complex Lie group isomorphism $\hat{\varphi}: G^{c} \rightarrow G^{\prime C}$ with $\hat{\varphi} G=G^{\prime}$ by

$$
\hat{\varphi}(x)=\varphi x \varphi^{-1} \quad \text { for } x \in G^{c} .
$$

The differential of $\hat{\varphi}$, denoted by the same $\hat{\varphi}: \mathfrak{g}^{c} \rightarrow \mathfrak{g}^{\prime c}$, is also an isomorphism with $\hat{\varphi} g=g^{\prime}$. Note that

$$
\hat{\varphi}(x) \circ \varphi=\varphi \circ x \quad \text { for each } x \in G^{c} .
$$

We define a homomorphism $\rho_{1}: G^{C} \rightarrow \mathrm{PL}(N+1, C)$ by

$$
\rho_{1}(x)=\Phi^{-1} \rho^{\prime}(\hat{\varphi}(x)) \Phi \quad \text { for } x \in G^{C} .
$$

Then, by (2.3), we have

$$
\begin{aligned}
\rho_{1}(x) f(p) & =\Phi^{-1} \rho^{\prime}(\hat{\varphi}(x)) \Phi f(p)=\Phi^{-1} \rho^{\prime}(\hat{\varphi}(x)) f^{\prime}(\varphi(p)) \\
& =\Phi^{-1} f^{\prime}(\hat{\varphi}(x) \varphi(p))=\Phi^{-1} f^{\prime}(\varphi(x p)) \\
& =\Phi^{-1} \Phi f(x p)=f(x p)
\end{aligned}
$$


for each $x \in G^{c}, p \in M$. The uniqueness of $\rho$ implies $\rho_{1}=\rho$, i.e.,

$$
\Phi^{-1} \rho^{\prime}(\hat{\varphi}(x)) \Phi=\rho(x) \quad \text { for each } x \in G^{c} .
$$

Taking an element $\tilde{\Phi} \in \mathrm{SU}(N+1)$ with $\pi(\tilde{\Phi})=\Phi$, we get

$$
\tilde{\Phi}^{-1} \rho^{\prime}(\hat{\varphi}(X)) \tilde{\Phi}=\rho(X) \quad \text { for each } X \in g^{c} .
$$

Choose $x_{1}^{\prime} \in G^{\prime}$ with $x_{1}^{\prime} \varphi(o)=o^{\prime}$. Then $U^{\prime}=x_{1}^{\prime} \hat{\varphi}(U) x_{1}^{\prime-1}$ and $K^{\prime}=x_{1}^{\prime} \hat{\varphi}(K) x_{1}^{\prime-1}$, and hence $\operatorname{Ad} x_{1}^{\prime} \hat{\varphi}(t)$ is a maximal abelian subalgebra of $\mathfrak{f}^{\prime}$. It follows that there exists $k_{1}^{\prime} \in K^{\prime}$ with $\mathrm{Ad} k_{1}^{\prime} \mathrm{Ad} x_{1}^{\prime} \hat{\varphi}(\mathrm{t})=\mathrm{t}^{\prime}$. The isomorphism Ad $\left(k_{1}^{\prime} x_{1}^{\prime}\right) \circ \hat{\varphi}: \mathrm{g}^{c} \rightarrow$ $\mathfrak{g}^{\prime C}$ satisfies $\left(\operatorname{Ad}\left(k_{1}^{\prime} x_{1}^{\prime}\right) \circ \hat{\varphi}\right) \Sigma=\Sigma^{\prime},\left(\operatorname{Ad}\left(k_{1}^{\prime} x_{1}^{\prime}\right) \circ \hat{\varphi}\right) \mathfrak{u}=\mathfrak{u}^{\prime}$ and $\left(\operatorname{Ad}\left(k_{1}^{\prime} x_{1}^{\prime}\right) \circ \hat{\varphi}\right) \mathfrak{f}=\mathfrak{l}^{\prime}$, and hence we have $\left(\operatorname{Ad}\left(k_{1}^{\prime} x_{1}^{\prime}\right) \circ \hat{\varphi}\right)\left(\Sigma_{0} \cup \Sigma^{+}\right)=\Sigma_{0}^{\prime} \cup \Sigma^{\prime+}$ and $\left(\operatorname{Ad}\left(k_{1}^{\prime} x_{1}^{\prime}\right) \circ \hat{\varphi}\right) \Sigma_{0}=\Sigma_{0}$. Thus we can find an element $k_{2}^{\prime} \in K^{\prime}$ which normalizes $t^{\prime}$ and satisfies $\left(\operatorname{Ad}\left(k_{2}^{\prime} k_{1}^{\prime} x_{1}^{\prime}\right) \circ \hat{\varphi}\right) \Pi=\Pi^{\prime}$ and $\left(\operatorname{Ad}\left(k_{2}^{\prime} k_{1}^{\prime} x_{1}^{\prime}\right) \circ \hat{\varphi}\right) \Pi_{0}=\Pi_{0}^{\prime}$. Putting $x_{0}^{\prime}=k_{2}^{\prime} k_{1}^{\prime} x_{1}^{\prime} \in G^{\prime}$, we define a complex Lie group isomorphism $\psi: G^{c} \rightarrow G^{\prime C}$ by

$$
\psi(x)=x_{0}^{\prime} \hat{\varphi}(x) x_{0}^{\prime-1} \quad \text { for } x \in G^{C} .
$$

The differential of $\psi$, denoted by the same $\psi: \mathfrak{g}^{c} \rightarrow \mathfrak{g}^{\prime c}$, is given by

$$
\psi(X)=\operatorname{Ad} x_{0}^{\prime} \hat{\varphi}(X) \quad \text { for } X \in \mathfrak{g}^{C} \text {. }
$$

Then they satisfy $\psi G=G^{\prime}, \psi U=U^{\prime}, \psi g=g^{\prime}, \psi t=t^{\prime}, \psi \Sigma=\Sigma^{\prime}, \psi \Pi=\Pi^{\prime}$ and $\psi \Pi_{0}=\Pi_{0}^{\prime}$. It follows from $x_{0} \varphi(o)=o^{\prime}$ that

$$
f^{\prime}\left(o^{\prime}\right)=f^{\prime}\left(x_{0}^{\prime} \varphi(o)\right)=\rho^{\prime}\left(x_{0}^{\prime}\right) f^{\prime}(\varphi(o))=\rho^{\prime}\left(x_{0}^{\prime}\right) \Phi f(o) .
$$

Hence, for a highest weight vector $v_{A}$ of $\rho$, the vector

$$
v_{\Lambda^{\prime}}^{\prime}=\widetilde{\rho^{\prime}\left(x_{0}^{\prime}\right)} \tilde{\Phi} v_{\Lambda}
$$

where $\widetilde{\rho^{\prime}\left(x_{0}^{\prime}\right)} \in \mathrm{SU}(N+1)$ with $\pi\left(\widetilde{\left.\rho^{\prime}\left(x_{0}^{\prime}\right)\right)}=\rho^{\prime}\left(x_{0}^{\prime}\right)\right.$, is a highest weight vector of $\rho^{\prime}$. Now, for each $H^{\prime} \in \mathfrak{t}^{\prime}$, we have

$$
\begin{aligned}
& \rho^{\prime}\left(H^{\prime}\right) v_{A^{\prime}}^{\prime}=\rho^{\prime}\left(H^{\prime}\right) \rho^{\prime}\left(x_{0}^{\prime}\right) \tilde{\Phi} v_{\Delta} \\
& =\widetilde{\rho^{\prime}\left(x_{0}^{\prime}\right)} \tilde{\Phi} \tilde{\Phi}^{-1} \rho^{\prime}\left(x_{0}^{\prime}\right)^{-1} \rho^{\prime}\left(H^{\prime}\right) \rho^{\prime}\left(x_{0}^{\prime}\right) \tilde{\Phi} v_{\Lambda}
\end{aligned}
$$

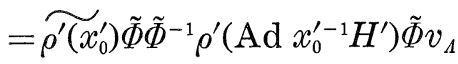

$$
\begin{aligned}
& =\widetilde{\rho^{\prime}\left(x_{0}^{\prime}\right)} \tilde{\Phi} \rho\left(\psi^{-1} H^{\prime}\right) v_{\Lambda} \quad \text { by (2.4), (2.5) }
\end{aligned}
$$

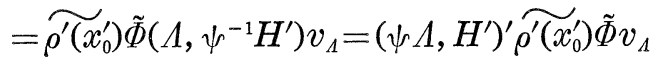

$$
\begin{aligned}
& =\left(\psi \Lambda, H^{\prime}\right)^{\prime} v_{\Lambda^{\prime}}^{\prime} \text {. }
\end{aligned}
$$


Therefore $\Lambda^{\prime}=\psi \Lambda$, and hence $p=p^{\prime} \circ \psi$ on $\Pi-\Pi_{0}$. Now $\psi$, restricted on $\Pi$, gives an equivalence $\psi^{\prime}:\left(\Pi, \Pi_{0} ; p\right) \rightarrow\left(\Pi^{\prime}, \Pi_{0}^{\prime} ; p^{\prime}\right)$.

q.e.d.

We give some examples of the correspondence in Theorem 2.2.

EXAMPLe 2.1. Let $\Pi$ be

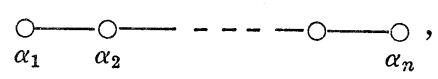

$\Pi-\Pi_{0}=\left\{\alpha_{1}\right\}$ and $p_{\alpha_{1}}=p \in Z^{+}$. Then $(M, g)=P_{n}\left(\frac{c}{p}\right)$ (This is seen by Theorem 4.3 in $\S 4), N+1=\left(\begin{array}{c}n+p \\ p\end{array}\right)$ and $f$ is given by

$$
f:\left[z_{i}\right]_{0 \leqslant i \leqslant n} \mapsto\left[\sqrt{\frac{p !}{p_{0} ! \cdots p_{n} !}} z_{0}^{p_{0}} \cdots z_{n}^{p_{n}}\right]_{p_{0}+\cdots+p_{n}=p},
$$

where $[*]$ means the point of the projective space with the homogeneous coordinates $*$. This is nothing but the example given by Calabi [4].

EXAMPLE 2.2. Let $\Pi$ be
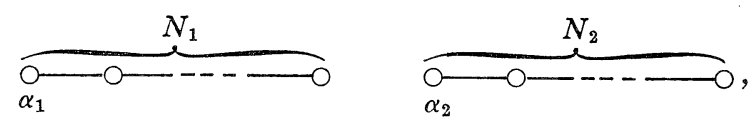

$\Pi-\Pi_{0}=\left\{\alpha_{1}, \alpha_{2}\right\}$ and $p_{\alpha_{1}}=p_{\alpha_{2}}=1$. Then $(M, g)=P_{N_{1}}(c) \times P_{N_{2}}(c), \quad N+1=$ $\left(N_{1}+1\right)\left(N_{2}+1\right)$ and $f$ is given by

$$
f:\left[z_{i}\right]_{0 \leqslant i \leqslant N_{1}} \times\left[w_{j}\right]_{0 \leqslant j \leqslant N_{2}} \mapsto\left[z_{i} w_{j}\right]_{\substack{0 \leqslant i \leqslant N_{1} \\ 0 \leqslant j \leqslant N_{2}}}
$$

This Kähler imbedding $f$ is called the tensor product of $P_{N_{1}}(c)$ and $P_{N_{2}}(c)$. The image $f\left(p_{1} \times p_{2}\right), p_{i} \in P_{N_{i}}(c)(i=1,2)$, will be denoted by $p_{1} \otimes p_{2}$.

REMARK 2.2. Let $\left(\Pi^{1}, \Pi_{0}^{1} ; p^{1}\right)$ and $\left(\Pi^{2}, \Pi_{0}^{2} ; p^{2}\right)$ be admissible triples. Put $\Pi=\Pi^{1} \cup \Pi^{2}, \Pi_{0}=\Pi_{0}^{1} \cup \Pi_{0}^{2}$ and define $p: \Pi-\Pi_{0} \rightarrow Z^{+}$by

$$
p(\alpha)= \begin{cases}p^{1}(\alpha) & \text { for } \alpha \in \Pi^{1}-\Pi_{0}^{1} \\ p^{2}(\alpha) & \text { for } \alpha \in \Pi^{2}-\Pi_{0}^{2} .\end{cases}
$$

Then $\left(\Pi, \Pi_{0} ; p\right)$ is also an admissible triple. It is called the direct product of $\left(\Pi^{1}, \Pi_{0}^{1} ; p^{1}\right)$ and $\left(\Pi^{2}, \Pi_{0}^{2} ; p^{2}\right)$, and denoted by $\left(\Pi^{1}, \Pi_{0}^{1} ; p^{1}\right) \times\left(\Pi^{2}, \Pi_{0}^{2} ; p^{2}\right)$.

Let $f_{1}:\left(M_{1}, g_{1}\right) \rightarrow P_{N_{1}}(c)$ and $f_{2}:\left(M_{2}, g_{2}\right) \rightarrow P_{N_{2}}(c)$ be Kähler immersions. The composition of the direct product $f_{1} \times f_{2}:\left(M_{1}, g_{1}\right) \times\left(M_{2}, g_{2}\right) \rightarrow P_{N_{1}}(c) \times$ $P_{N_{2}}(c)$ and the tensor product: $P_{N_{1}}(c) \times P_{N_{2}}(c) \rightarrow P_{N}(c), N+1=\left(N_{1}+1\right)\left(N_{2}+1\right)$, is called the tensor product of $f_{1}$ and $f_{2}$, and denoted by $f_{1} \otimes f_{2}:\left(M_{1}, g_{1}\right) \times$ $\left(M_{2}, g_{2}\right) \rightarrow P_{N}(c)$. It is also a Kähler immersion and satisfies 


$$
\left(f_{1} \otimes f_{2}\right)\left(p_{1} \times p_{2}\right)=f_{1}\left(p_{1}\right) \otimes f_{2}\left(p_{2}\right) \quad \text { for } p_{1} \in M_{1}, p \in M_{2} \text {. }
$$

With these definitions, we have the following: Let $\left(\Pi^{1}, \Pi_{0}^{1} ; p^{1}\right)$ and $\left(\Pi^{2}, \Pi_{0}^{2} ; p^{2}\right)$ be admissible triples, and let $f_{1}:\left(M_{1}, g_{1}\right) \rightarrow P_{N_{1}}(c)$ and $f_{2}:\left(M_{2}, g_{2}\right)$ $\rightarrow P_{N_{2}}(c)$ be the full Kähler immersions associated to $\left(\Pi^{1}, \Pi_{0}^{1} ; p^{1}\right)$ and $\left(\Pi^{2}, \Pi_{0}^{2}\right.$; $\left.p^{2}\right)$ respectively. Then the full Kähler immersion associated to the direct product $\left(\Pi^{1}, \Pi_{0}^{1} ; p^{1}\right) \times\left(\Pi^{2}, \Pi_{0}^{2} ; p^{2}\right)$ is the tensor product $f_{1} \otimes f_{2}$ of $f_{1}$ and $f_{2}$.

Thus, each full Kähler immersion $f:(M, g) \rightarrow P_{N}(c)$ of a homogeneous Kähler manifold $(M, g)$ is the tensor product:

$$
f=f_{1} \otimes \cdots \otimes f_{s}:(M, g)=\left(M_{1}, g_{1}\right) \times \cdots \times\left(M_{s}, g_{s}\right) \rightarrow P_{N}(c),
$$

of full Kähler immersions $f_{i}:\left(M_{i}, g_{i}\right) \rightarrow P_{N_{i}}(c)(1 \leqslant i \leqslant s)$ of homogeneous Kähler manifolds $\left(M_{i}, g_{i}\right)$ on which compact connected simple Lie groups $G_{i}$ act transitively, since each admissible triple $\left(\Pi, \Pi_{0} ; p\right)$ has the direct product decomposition:

$$
\left(\Pi, \Pi_{0}, p\right)=\left(\Pi^{1}, \Pi_{0}^{1} ; p^{1}\right) \times \cdots \times\left(\Pi^{s}, \Pi_{0}^{s} ; p^{s}\right),
$$

with $\Pi^{i}$ irreducible. Note that the decomposition:

$$
(M, g)=\left(M_{1}, g_{1}\right) \times \cdots \times\left(M_{s}, g_{s}\right)
$$

is the de Rham decomposition of the Kähler manifold $(M, g)$ and that each $\operatorname{Aut}^{0}\left(M_{i}, g_{i}\right)$ is also simple (cf. Hano-Matsushima [6]).

REMARK 2.3. The dimension $N$ of the target Fubini-Study space of the full Kähler immersion associated to an admissible triple $\left(\Pi, \Pi_{0} ; p\right)$ is given by the Weyl's formula:

$$
N+1=\prod_{\alpha \in \Sigma^{+}} \frac{(\Lambda+\delta, \alpha)}{(\delta, \alpha)}
$$

where

$$
\Lambda=\sum_{\alpha \in \Pi=\Pi_{0}} p_{\alpha} \Lambda_{\alpha}, \quad \delta=\frac{1}{2} \sum_{\alpha \in \Sigma+} \alpha .
$$

REMARK 2.4. The correspondence in Theorem 2.2 is not injective. For example, let $\Pi$ be

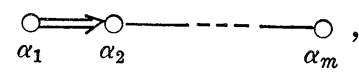

$\Pi-\Pi_{0}=\left\{\alpha_{1}\right\}$ and $p_{\alpha_{1}}=p \in Z^{+} . \quad$ Then, $(M, g)=P_{2 m-1}\left(\frac{c}{p}\right)$ and $f$ is equivalent 
to the Kähler imbedding in Example 2.1, with $n=2 m-1$.

REMARK 2.5. Theorem 2.2 was given by Nakagawa-Takagi [15] in a weaker form for the case where $M$ is a compact simply connected homogeneous Kähler manifold with $b_{2}(M)=1$.

\section{§ 3. Homogeneous Kähler Einstein submanifolds of $\boldsymbol{P}_{N}(\boldsymbol{c})$}

In this section, we shall compute certain geometric invariants of homogeneous Kähler submanifolds of $P_{N}(c)$ and find all homogeneous Kähler Einstein submanifolds of $P_{N}(c)$.

Let $M=G^{c} / U=G / K$ be the homogeneous complex manifold associated to an effective pair $\left(\Pi, \Pi_{0}\right)$ of Dynkin diagrams as in $\S 2$. We retain the notation in $\S 2$. We denote by $X \mapsto \bar{X}$ the complex conjugation of $\mathrm{g}^{c}$ with respect to the real form $g$. We define a subspace $c$ of $\sqrt{-1} t$ by

$$
\mathfrak{c}=\sum_{\alpha \in \Pi-\Pi_{0}} R \Lambda_{\alpha}
$$

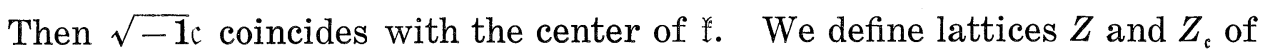
$\sqrt{-1}$ t and $c$ respectively by

$$
Z=\left\{\lambda \in \sqrt{-1} t ; \frac{2(\lambda, \alpha)}{(\alpha, \alpha)} \in Z \text { for each } \alpha \in \Sigma\right\}
$$

and

$$
Z_{\mathrm{c}}=Z \cap \mathrm{c}
$$

We define a cone $c^{+}$in $c$ by

$$
\mathfrak{c}^{+}=\left\{\lambda \in \mathfrak{c} ;(\lambda, \alpha)>0 \text { for each } \alpha \in \Pi-\Pi_{0}\right\},
$$

and put

$$
Z_{\mathrm{c}}^{+}=Z \cap \mathrm{c}^{+}
$$

Then we have

$$
\mathfrak{c}^{+}=\sum_{\alpha \in \Pi-\Pi_{0}} \boldsymbol{R}^{+} \Lambda_{\alpha}
$$

and

$$
Z_{\mathrm{c}}^{+}=\sum_{\alpha \in \Pi-\Pi_{0}} Z^{+} \Lambda_{\alpha}
$$

where $\boldsymbol{R}^{+}$and $Z^{+}$denote the set of positive reals and the one of positive 
integers respectively. Let $\mathfrak{m}$ be the orthogonal complement of $\mathfrak{i n} \mathrm{g}$ with respect to the Killing form (, ). Then we have a direct sum decomposition:

$$
\mathfrak{g}=\mathfrak{i}+\mathfrak{m}
$$

as vector spaces. The subspace $\mathfrak{m}$ is $K$-invariant (under the adjoint action) and identified with the tangent space $T_{o} M$ of $M$ at the origin. Put

$$
\Sigma_{\mathfrak{m}}^{+}=\Sigma^{+}-\Sigma_{0}, \quad \Sigma_{\mathfrak{m}}^{-}=-\Sigma_{\mathfrak{m}}^{+},
$$

and define $K$-invariant subspaces $\mathfrak{m}^{ \pm}$of $\mathrm{g}^{c}$ by

$$
\mathfrak{m}^{ \pm}=\sum_{\alpha \in \Sigma_{\mathfrak{m}}^{ \pm}} \mathfrak{g}_{-\alpha}^{C} \text {. }
$$

Then $\overline{\mathfrak{m}}^{ \pm}=\mathfrak{m}^{\mp}$ and the complexification $\mathfrak{m}^{c}$ of $\mathfrak{n}$ is the direct sum :

$$
\mathfrak{m}^{c}=\mathfrak{m}^{+}+\mathfrak{m}^{-} \text {. }
$$

Note that the cone $\mathrm{c}^{+}$is characterized by

$$
\mathfrak{c}^{+}=\left\{\lambda \in \mathfrak{c} ;(\lambda, \alpha)>0 \text { for each } \alpha \in \Sigma_{\mathfrak{m}}^{+}\right\} .
$$

We choose $E_{\alpha} \in \mathrm{g}_{\alpha}^{C}$ for $\alpha \in \Sigma$ with the following properties and fix them once for all :

$$
\left[E_{\alpha}, E_{-\alpha}\right]=-\alpha, \quad\left(E_{\alpha}, E_{-\alpha}\right)=-1, \quad \bar{E}_{\alpha}=E_{-\alpha} \quad \text { for } \alpha \in \Sigma \text {. }
$$

Let $\left\{\omega^{\alpha}\right\}_{\alpha \in \Sigma}$ be the linear forms on $\mathrm{g}^{c}$ dual to $\left\{E_{\alpha}\right\}_{\alpha \in \Sigma}$, more precisely, the linear forms defined by

$$
\begin{aligned}
\omega^{\alpha}\left(t^{c}\right) & =\{0\}, \\
\omega^{\alpha}\left(E_{\beta}\right) & = \begin{cases}1 & \text { if } \alpha=\beta \\
0 & \text { if } \alpha \neq \beta .\end{cases}
\end{aligned}
$$

Let $\tilde{U}$ be the connected (closed) complex subgroup of $\tilde{G}^{c}$ generated by $\mathfrak{u}$ and put $\tilde{K}=\tilde{G} \cap \tilde{U}$. Then we have also identifications: $\quad M=\tilde{G}^{c} / \tilde{U}=\tilde{G} / \tilde{K}$. Let $\tilde{T}$ be the toral subgroup of $\tilde{G}$ generated by t. The tangent space $T_{e} \tilde{T}$ of $\tilde{T}$ at $e$ is identified with $t$ and so the cotangent space $T_{e}^{*} \tilde{T}$ with the dual space $t^{*}$ of $t$. Let $\mathscr{I}^{1}(\tilde{T})$ denote the space of $\tilde{T}$-invariant real 1 -forms on $\tilde{T}$. Then we have a series of linear isomorphisms:

$$
\mathrm{t} \rightarrow \mathrm{t}^{*}=T_{e}^{*} \tilde{T} \rightarrow \mathscr{I}^{1}(\tilde{T}) \rightarrow H^{1}(\tilde{T}, R),
$$

where the first one is the duality defined by the Killing form (, ) and the 
second and the third are the natural isomorphisms. In what follows, we shall identify $\downarrow$ with $H^{1}(\tilde{T}, R)$ by means of the composition of these isomorphisms. We have then an identification (cf. Helgason [7]) :

$$
\frac{1}{2 \pi \sqrt{-1}} Z=H^{1}(\tilde{T}, Z)
$$

It is known (Borel-Hirzebruch [1]) that the injection $\iota: \tilde{T}_{\subset} \longrightarrow \tilde{K}$ induces an injective linear map $\iota^{*}: H^{1}(\tilde{K}, R) \rightarrow H^{1}(\tilde{T}, R)$ with $\iota^{*}\left(H^{1}(\tilde{K}, R)\right)=\frac{1}{2 \pi \sqrt{-1}} c$ and $\iota^{*}\left(H^{1}(\tilde{K}, Z)\right)=\frac{1}{2 \pi \sqrt{-1}} Z_{\mathrm{c}}$, and that the transgression operator for the principal bundle $\tilde{K} \rightarrow \tilde{G} \rightarrow M$ defines a linear isomorphism $\tau: H^{1}(\tilde{K}, R) \rightarrow H^{2}(M, R)$ with $\tau\left(H^{1}(\tilde{K}, Z)\right)=H^{2}(M, Z)$. Thus, if we define a linear map $\tau: \mathcal{c} \rightarrow H^{2}(M, R)$ by

$$
\tau(\lambda)=-\tau\left(\frac{\lambda}{2 \pi \sqrt{-1}}\right) \quad \text { for } \lambda \in \mathfrak{c}
$$

identifying $H^{1}(\tilde{K}, \boldsymbol{R})$ with a subspace of $H^{1}(\tilde{T}, \boldsymbol{R})$ through $\iota^{*}$, then $\tau$ is a linear isomorphism such that the image $\tau\left(Z_{\mathrm{c}}\right)$ of $Z_{\mathrm{c}}$ coincides with $H^{2}(M, Z)$. Note that in the above arguments we have identified $H^{1}(\tilde{T}, Z), H^{1}(\tilde{K}, Z)$ and $H^{2}(M, Z)$ with subgroups of $H^{1}(\tilde{T}, \boldsymbol{R}), H^{1}(\tilde{K}, \boldsymbol{R})$ and $H^{2}(M, \boldsymbol{R})$ respectively. This can be done since these integral cohomology groups have no torsion (cf. Borel-Hirzebruch [1]).

LEMMA 3.1. Let $\mathscr{I}_{G}^{2}(M)$ be the space of closed G-invariant real 2-forms on $M$ and $\mathscr{H}^{2}(M, g)$ the space of real harmonic 2 -forms on $M$ with respect to a G-invariant Riemannian metric $g$ on $M$. Then

$$
\mathscr{I}_{G}^{2}(M)=\mathscr{H}^{2}(M, g) \text {. }
$$

Proof. First we shall prove that there is no $G$-invariant real 1-form on $M$. For this purpose, it suffices to show that if $\omega$ is a $K$-invariant complex linear form on $\mathrm{m}^{+}$, then $\omega=0$. By means of the duality by the Killing form, $\omega$ is identified with an element $X_{\omega} \in \mathfrak{m}^{-}$with $\left[\mathfrak{f}, X_{\omega}\right]=\{0\}$. But each element $H \in \mathfrak{c}^{+}$induces a non-singular endomorphism on $\mathrm{m}^{-}$. Therefore $X_{\omega}=0$ and hence $\omega=0$.

Let $d^{*}$ denote the formal adjoint operator of $d$ with respect to the Riemannian measure defined by $g$. Then, for each $\eta \in \mathscr{I}_{G}^{2}(M), d^{*} \eta$ is a $G$ invariant real 1 -form on $M$. It follows from the above that $d^{*} \eta=0$, and hence $\eta \in \mathscr{H}^{2}(M, g)$. This proves $\mathscr{I}_{G}^{2}(M) \subset \mathscr{H}^{2}(M, g)$. Together with the surjectivity of the natural map $\widetilde{\sigma}: \mathscr{I}_{G}^{2}(M) \rightarrow H^{2}(M, R)$ (Chevalley-Eilenberg 
[5]), we conclude $\mathscr{I}_{G}^{2}(M)=\mathscr{H}^{2}(M, g)$.

q.e.d.

Let $\lambda \in \mathfrak{c}$. Regarding each $\omega^{\alpha}$ a $\tilde{G}$-invariant $C$-valued 1 -form on $\tilde{G}$, we define a $\tilde{G}$-invariant $C$-valued 2 -form $\eta(\lambda)$ on $\tilde{G}$ by

$$
\eta(\lambda)=-\frac{\sqrt{-1}}{2 \pi} \sum_{\alpha \in \Sigma_{\mathfrak{m}}^{+}}(\lambda, \alpha) \omega^{-\alpha} \wedge \bar{\omega}^{-\alpha} .
$$

On the other hand, we define a complex linear form $\tilde{\lambda}$ on $\mathfrak{g}$ by

$$
\tilde{\lambda}(X)=(\lambda, X) \quad \text { for } X \in \mathfrak{g},
$$

and regard $\tilde{\lambda}$ a $\tilde{G}$-invariant $C$-valued 1 -form on $\tilde{G}$, thus $\frac{1}{2 \pi \sqrt{-1}} \tilde{\lambda}$ a $\tilde{G}$-invariant $R$-valued 1 -form on $\tilde{G}$. Then a straightforward calculation shows

$$
\eta(\lambda)=-d\left(\frac{1}{2 \pi \sqrt{-1}} \tilde{\lambda}\right)
$$

It follows from $[f, c]=\{0\}$ and the definition (3.1) of $\eta(\lambda)$ that $\eta(\lambda)$ is a $\tilde{G}$-invariant $\boldsymbol{R}$-valued 1 -form on $\tilde{G}$ satisfying

$$
\begin{array}{ll}
R_{k}^{*} \eta(\lambda)=\eta(\lambda) & \text { for each } k \in \tilde{K}, \\
i(X) \eta(\lambda)=0 & \text { for each } X \in \mathfrak{l},
\end{array}
$$

where $R_{k}$ means the right translation in $\tilde{G}$ by $k$, and $i(X)$ the contraction by $X$. Hence $\eta(\lambda)$ is pulled down to a unique form in $\mathscr{I}_{G}^{2}(M)$. In what follows, we shall identify $\eta(\lambda)$ with this form in $\mathscr{I}_{G}^{2}(M)$, and hence the correspondence $\lambda \mapsto \eta(\lambda)$ defines a linear $\operatorname{map} \eta: \mathfrak{c} \rightarrow \mathscr{I}_{G}^{2}(M)$.

LEMMA 3.2. We have the following commutative diagram consisting of linear isomorphisms:

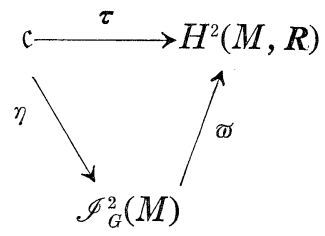

where $\widetilde{w}$ is the natural map assigning to $\omega \in \mathscr{I}_{G}^{2}(M)$ the de Rham class $[\omega] \in$ $H^{2}(M, R)$ of $\omega$.

Proof. The commutativity follows from the definition of the transgression and (3.2). The natural map $\varpi$ is an isomorphism by Lemma 3.1, and hence $\eta$ is also an isomorphism.

q.e.d. 
We define an element $\delta_{\mathfrak{m}} \in \sqrt{-1} \mathrm{t}$ by

$$
\delta_{\mathfrak{m}}=\frac{1}{2} \sum_{\alpha \in \Sigma_{\mathfrak{m}}^{+}} \alpha .
$$

Let $c_{1}(M) \in H^{2}(M, Z)$ denote the first Chern class of $M$. It is then known (Borel-Hirzebruch [1]) that

$$
\begin{gathered}
2 \delta_{\mathfrak{m}} \in Z_{\mathfrak{c}}^{+}, \\
c_{1}(M)=-\tau\left(2 \delta_{\mathfrak{m}}\right) .
\end{gathered}
$$

THEOREM 3.1. Let $M=G^{c} / U=G / K$ be the homogeneous complex manifold associated to an effective pair $\left(\Pi, \Pi_{0}\right)$. Then

1) (Borel-Hirzebruch [1]). For $\lambda \in \mathfrak{c}$,

$$
g(\lambda)=\frac{1}{2 \pi} \sum_{\alpha \in \Sigma_{\mathfrak{m}}^{+}}(\lambda, \alpha) \omega^{-\alpha} \cdot \bar{\omega}^{-\alpha}
$$

defines a G-invariant real covariant symmetric tensor field of degree 2 on $M$, and the correspondence $\lambda \mapsto g(\lambda)$ gives a bijection from $c^{+}$onto the set of $G$-invariant Kähler metrics on $M$.

2) For the Kähler metric $g$ corresponding to $\lambda \in \mathfrak{c}^{+}$, the Kähler form $\Omega$ (defined by $\Omega(X, Y)=g(X, J Y), J$ being the complex structure tensor of $M$ ), the Ricci tensor $S$, the Ricci form $\sigma$ (defined by $\sigma(X, Y)=S(X, J Y)$ ) and the scalar curvature $k$ are given by

$$
\begin{aligned}
& \Omega=\eta(\lambda)=-\frac{\sqrt{-1}}{2 \pi} \sum_{\alpha \in \Sigma_{\mathfrak{m}}^{+}}(\lambda, \alpha) \omega^{-\alpha} \wedge \bar{\omega}^{-\alpha}, \\
& S=8 \pi g\left(\delta_{\mathfrak{m}}\right)=4 \sum_{\alpha \in \Sigma_{\mathfrak{m}}^{+}}\left(\delta_{\mathfrak{m}}, \alpha\right) \omega^{-\alpha} \cdot \bar{\omega}^{-\alpha}, \\
& \sigma=8 \pi \eta\left(\delta_{\mathfrak{m}}\right)=-4 \sqrt{-1} \sum_{\alpha \in \Sigma_{\mathfrak{m}}^{+}}\left(\delta_{\mathfrak{m}}, \alpha\right) \omega^{-\alpha} \wedge \bar{\omega}^{-\alpha}, \\
& k=16 \pi \sum_{\alpha \in \Sigma_{\mathfrak{m}}^{+}} \frac{\left(\delta_{\mathfrak{m}}, \alpha\right)}{(\lambda, \alpha)} .
\end{aligned}
$$

Thus $S$ and $\sigma$ are independent of $g$.

Proof. For $\lambda \in \mathfrak{c}$, we define a $G$-invariant covariant tensor field $g(\lambda)$ of degree 2 on $M$ by

$$
g(\lambda)(X, Y)=\eta(\lambda)(J X, Y) \quad \text { for smooth vector fields } X, Y \text { on } M \text {. }
$$

Then the pull back of $g(\lambda)$ to $\tilde{G}$ has the expression as the right hand side of 
(3.5). This proves the first assertion of 1 ). If $g$ is a $G$-invariant Kähler metric on $M$, then the Kähler form $\Omega$ for $g$ is in $\mathscr{I}_{G}^{2}(M)$. It follows from Lemma 3.2 that it is written as $\Omega=\eta(\lambda)$ by an element $\lambda \in \mathfrak{c}$, and hence $g=$ $g(\lambda)$. The positive definiteness of $g$ implies $\lambda \in \mathfrak{c}^{+}$. This proves the second assertion of 1) and the first formula in 2).

It is known (cf. Kobayashi-Nomizu [10], Chap. XII) that the Ricci form $\sigma$ is closed and the real first Chern class $c_{1}(M)_{R}$ is given by

$$
c_{1}(M)_{R}=-\frac{1}{4 \pi}[\sigma]
$$

for a general Kähler manifold $(M, g)$. In our case, $\sigma \in \mathscr{I}_{G}^{2}(M)$, and from (3.4) and Lemma 3.2 we have

$$
\widetilde{\sigma}(\sigma)=8 \pi \tau\left(\delta_{\mathfrak{m}}\right)=\varpi\left(8 \pi \eta\left(\delta_{\mathfrak{m}}\right)\right) .
$$

Thus, by Lemma 3.2, we get the formula for $\sigma$ :

$$
\sigma=8 \pi \eta\left(\delta_{\mathfrak{m}}\right)
$$

The formula for $S$ is a consequence of the one for $\sigma$.

To compute $k$, let

$$
e_{\alpha}=2 \sqrt{\frac{\pi}{(\lambda, \alpha)}} E_{-\alpha} \quad \text { for } \alpha \in \sum_{\mathfrak{m}}^{+} .
$$

Then, extending $g$ and $S C$-bilinearly to $\mathrm{m}^{c}$, we get

$$
\begin{aligned}
& g\left(e_{\alpha}, \bar{e}_{\beta}\right)=\delta_{\alpha \beta}, \\
& S\left(e_{\alpha}, \bar{e}_{\beta}\right)=8 \pi \frac{\left(\delta_{m}, \alpha\right)}{(\lambda, \alpha)} \delta_{\alpha \beta},
\end{aligned}
$$

and hence

$$
k=2 \sum_{\alpha \in \Sigma_{\mathfrak{m}}^{+}} S\left(e_{\alpha}, \bar{e}_{\alpha}\right)=16 \pi \sum_{\alpha \in \Sigma_{\mathfrak{m}}^{+}} \frac{\left(\delta_{\mathfrak{m}}, \alpha\right)}{(\lambda, \alpha)} .
$$

CoRollaRY. The G-invariant Kähler metric $g$ on $M$ corresponding to $\lambda \in \mathfrak{c}^{+}$is Einstein if and only if there exists $b>0$ such that $\lambda=b \delta_{\mathrm{m}}$. In this case,

$$
\begin{aligned}
& S=\frac{8 \pi}{b} g, \\
& k=\frac{16 \pi n}{b}, \quad \text { where } n=\operatorname{dim}_{C} M .
\end{aligned}
$$


Proof. $g$ is Einstein if and only if $S=a g$ for some $a \in R$. From the Theorem, 2), the relation $S=a g$ is equivalent to $4 \delta_{\mathrm{m}}=\frac{a}{2 \pi} \lambda$. Since both $\delta_{\mathrm{m}}$ and $\lambda$ belong to $c^{+}$by (3.3), the latter relation is equivalent to $\lambda=b \delta_{\mathfrak{m}}$ with $a b=8 \pi, b>0$. This and the Theorem, 2) prove the assertions. q.e.d.

REMARK 3.1. Let $(M, g)$ be a compact simply connected homogeneous Kähler manifold. Then, for any connected Lie subgroup $G$ of $\operatorname{Aut}^{0}(M, g)$ acting transitively on $M$, the set of $G$-invariant Kähler metrics on $M$ coincides with the one of $\operatorname{Aut}^{0}(M, g)$-invariant Kähler metrics on $M$. This is verified as follows.

Note first that $\operatorname{dim} \mathscr{I}_{\text {Auto }(M, g)}^{2}(M)$ is equal to the second Betti number $b_{2}(M)$ of $M$, by Lemma 3.1. On the other hand, by Theorem M, $G$ is a compact connected semi-simple Lie group without center. Let $\mathrm{g}^{c}$ be the complexification of the Lie algebra $g \subset a(M)$ of $G$ and $G^{c}$ the connected complex Lie subgroup of $\operatorname{Aut}^{0}(M)$ generated by $\mathrm{g}^{c}$. Denoting the stabilizers in $G$ and in $G^{C}$ of a point of $M$ by $K$ and $U$ respectively, we have identifications: $M=G / K=G^{c} / U$. Then, as in the part (ii) of the proof of Theorem 2.1 , we have an effective pair $\left(\Pi, \Pi_{0}\right)$ of Dynkin diagrams, to which $M=$ $G / K=G^{c} / U$ is associated. Therefore, by Lemma 3.1, we have also $\operatorname{dim} \mathscr{I}_{G}^{2}(M)=b_{2}(M)$. Since $\mathscr{I}_{\text {Aut }(M, g)}^{2}(M) \subset \mathscr{I}_{G}^{2}(M)$, we get

$$
\mathscr{I}_{\mathrm{Aut}(M, g)}^{2}(M)=\mathscr{I}_{G}^{2}(M) \text {. }
$$

Recalling the correspondence between Kähler metrics and Kähler forms, we get a proof of the assertion.

THEOREM 3.2. Let $f:(M, g) \rightarrow P_{N}(c)$ be a full Kähler immersion of a homogeneous Kähler manifold $(M, g)$ associated to an admissible triple $\left(\Pi, \Pi_{0} ; p\right) . \quad$ Put

$$
\Lambda=\sum_{\alpha \in \Pi-\Pi_{0}} p_{\alpha} \Lambda_{\alpha} \in Z_{\mathrm{c}}^{+} \subset \mathfrak{c}^{+}
$$

Then the Kähler metric $g$ is given by

$$
g=g\left(\frac{8 \pi}{c} \Lambda\right)=\frac{4}{c} \sum_{\alpha \in \Sigma_{\mathfrak{m}}^{+}}(\Lambda, \alpha) \omega^{-a} \cdot \bar{\omega}^{-\alpha}
$$

with the notation in Theorem 3.1. Thus (by Theorem 3.1) the scalar curvature $k$ is given by

$$
k=2 c \sum_{\alpha \in \Sigma_{\mathfrak{m}}^{+}} \frac{\left(\delta_{\mathfrak{m}}, \alpha\right)}{(\Lambda, \alpha)}
$$


Proof. It suffices to show that the Kähler form $\Omega$ for $g$ satisfies

$$
\Omega=\frac{8 \pi}{c} \eta(\Lambda)
$$

Let $E_{N}$ be the canonical line bundle over $P_{N}(C)$. The fibre $\left(E_{N}\right)_{[v]}$ over $[v] \in P_{N}(C), v \in C^{N+1}-\{0\}$, may be considered as the 1-dimensional subspace $C v$ of $C^{N+1}$ spanned by $v$. It is also described as a homogeneous vector bundle as follows. Let

$$
\begin{aligned}
\mathrm{SL}(1, N ; C) & =\left\{\left(\frac{\alpha}{c \mid} \mid \frac{b}{d}\right) \in \mathrm{SL}(N+1, C) ; \alpha \in C^{*}, c=0\right\}, \\
\mathrm{SU}(1, N) & =\mathrm{SU}(N+1) \cap \mathrm{SL}(1, N ; C),
\end{aligned}
$$

and define a holomorphic representation $\lambda_{1}: \mathrm{SL}(1, N ; C) \rightarrow \mathrm{GL}(1, C)=C^{*}$ by

$$
\lambda_{1}:\left(\begin{array}{cc}
\alpha & b \\
0 & d
\end{array}\right) \mapsto \alpha
$$

Then the map $x \times z \mapsto x\left(\begin{array}{c}z \\ 0 \\ \vdots \\ 0\end{array}\right)(x \in \mathrm{SL}(N+1, C z \in C)$ induces the identification of $E_{N}$ with the homogeneous vector bundle $\mathrm{SL}(N+1, C) \underset{\mathrm{SL}(1, N ; C)}{\times} C$ over $P_{N}(C)=\mathrm{SL}(N+1, C) / \mathrm{SL}(1, N ; C)$ associated to the representation $\lambda_{1}$. $E_{N}$ is also identified, as a smooth vector bundle, with the homogeneous vecor bundle $\mathrm{SU}(N+1) \underset{\mathrm{SU}(N, 1)}{\times} C$ over $P_{N}(C)=\mathrm{SU}(N+1) / \mathrm{SU}(1, N)$ associated to the restricted representation $\lambda_{1}: \mathrm{SU}(1, N) \rightarrow U(1)$. It is known (Kobayashi-Nomizu [10], Chap. IX and $X I I$ ) that the first Chern class $c_{1}\left(E_{N}\right)$ of $E_{N}$ is related to the Kähler form $\Omega_{N}$ of $P_{N}(c)$ by

$$
c_{1}\left(E_{N}\right)=\frac{c}{8 \pi}\left[\Omega_{N}\right]
$$

Consider the pull back bundle $f^{*} E_{N}$ of $E_{N}$. Since we may assume that the associated representation $\tilde{\rho}: \tilde{G}^{c} \rightarrow \mathrm{SL}(N+1, C)$ with $\tilde{\rho}(\tilde{G}) \subset \mathrm{SU}(N+1)$ satisfies $\tilde{\rho}(\tilde{U}) \subset \mathrm{SL}(1, N ; C)$, we have an identification:

$$
f^{*} E_{N}=\tilde{G}^{c} \times_{\tilde{U}} C
$$

where $\tilde{U}$ acts on $C$ by the representation $\lambda_{1} \circ \tilde{\rho}: \tilde{U} \rightarrow \mathrm{GL}(1, C)$. This representation $\lambda_{1} \circ \tilde{\rho}$ of $\tilde{U}$ is characterized by 


$$
\left(\lambda_{1} \circ \tilde{\rho}\right)(\exp H)=e^{(\Lambda, H)} \quad \text { for each } H \in \mathfrak{t}^{c} .
$$

We have also an identification as a smooth vector bundle :

$$
f^{*} E_{N}=\tilde{G} \times_{\tilde{K}} C,
$$

where $\tilde{K}$ acts on $C$ by the restricted representation $\lambda_{1} \circ \tilde{\rho}: \tilde{K} \rightarrow U(1)$. It follows (Borel-Hirzebruch [1]) that $c_{1}\left(f^{*} E_{N}\right)=\tau(\Lambda)$, and hence, by Lemma 3.2,

$$
\begin{aligned}
\widetilde{\varpi}\left(\frac{c}{8 \pi} \Omega\right) & =\frac{c}{8 \pi}[\Omega]=\frac{c}{8 \pi}\left[f^{*} \Omega_{N}\right]=f^{*} c_{1}\left(E_{N}\right)=c_{1}\left(f^{*} E_{N}\right) \\
& =\tau(\Lambda)=\varpi(\eta(\Lambda)) .
\end{aligned}
$$

Thus, by Lemma 3.2, we get

$$
\frac{c}{8 \pi} \Omega=\eta(\Lambda)
$$

which implies the required equality.

q.e.d.

Put

$$
k_{\alpha}=k_{\alpha}(M)=\sum_{\beta \in \Sigma_{\mathfrak{m}}^{+}} \frac{2(\beta, \alpha)}{(\alpha, \alpha)}=\frac{4\left(\delta_{\mathfrak{m}}, \alpha\right)}{(\alpha, \alpha)} \quad \text { for } \alpha \in \Pi-\Pi_{0} .
$$

Then

$$
2 \delta_{\mathfrak{m}}=\sum_{\alpha \in \Pi I-\Pi_{0}} k_{\alpha} \Lambda_{\alpha}
$$

and each $k_{\alpha}$ is a positive integer by (3.3). Let $\kappa=\kappa(M)$ be the greatest common divisor of $\left\{k_{\alpha}\right\}_{\alpha \in \Pi-\Pi_{0}}$, and put

$$
\begin{gathered}
\kappa_{\alpha}=\kappa_{\alpha}(M)=\frac{k_{\alpha}}{\kappa} \quad \text { for } \alpha \in \Pi-\Pi_{0}, \\
g_{M}=\frac{8}{\kappa(M)} \sum_{\alpha \in \Sigma_{m}^{+}}\left(\delta_{\mathfrak{m}}, \alpha\right) \omega^{-\alpha} \cdot \bar{\omega}^{-\alpha} .
\end{gathered}
$$

With these notations, we can characterize full Kähler Einstein immersions in the following

COROLLARY. The Kähler metric $g$ in the Theorem is Einstein if and only if there exists $p \in Z^{+}$with

$$
p_{\alpha}=p \kappa_{\alpha}(M) \quad \text { for each } \alpha \in \Pi-\Pi_{0} .
$$

In this case, the Einstein Kähler metric g, the Ricci tensor $S$ and the scalar curvature k are given by 


$$
\begin{aligned}
g & =\frac{p}{c} g_{M}, \\
S & =\frac{c \kappa(M)}{2 p} g, \\
k & =\frac{n c \kappa(M)}{p}, \quad \text { where } n=\operatorname{dim}_{C} M .
\end{aligned}
$$

Proof. By Corollary of Theorem 3.1, $g$ is Einstein if and only if there exists $b^{\prime}>0$ with $\Lambda=b^{\prime}\left(2 \delta_{\mathrm{m}}\right)$. But since

$$
\begin{array}{cc}
\Lambda=\sum_{\alpha \in \Pi-\Pi_{0}} p_{\alpha} \Lambda_{\alpha}, & p_{\alpha} \in Z^{+}, \\
2 \delta_{\mathfrak{m}}=\sum_{\alpha \in \Pi-\Pi_{0}} k_{\alpha} \Lambda_{\alpha}, & k_{\alpha} \in Z^{+},
\end{array}
$$

the above condition is equivalent to the one stated in the Corollary. In this case, $\frac{8 \pi}{c} \Lambda=b \delta_{\mathfrak{m}}$ with $b=\frac{16 \pi}{c \kappa} p$. Thus, by Corollary of Theorem 3.1, we get the formulas for $S$ and $k$. The formula for $g$ follows from the Theorem and the relation: $\Lambda=\frac{2 p}{\kappa} \delta_{\mathfrak{m}}$.

q.e.d.

THEOREM 3.3. Let $(M, g)$ be a homogeneous Kähler manifold which admits a Kähler immersion into a Fubini-Study space. Let $\left(\Pi, \Pi_{0}\right)$ be the effective pair associated to $M=G / K=G^{c} / U$, where $G=\operatorname{Aut}^{0}(M, g)$ and $G^{c}$ $=\operatorname{Aut}^{0}(M)$, as in Theorem 2.2 (ii). With the notation in Theorem 2.2 (ii), we define a finite group Aut $\left(\Pi, \Pi_{0}\right)$ by

$$
\operatorname{Aut}\left(\Pi, \Pi_{0}\right)=\left\{\psi \in \mathrm{GL}(\sqrt{-1} \mathrm{t}) ; \psi \Sigma=\Sigma, \psi \Pi=\Pi \text { and } \psi \Pi_{0}=\Pi_{0}\right\},
$$

which acts on the cone

$$
\mathfrak{c}^{+}=\sum_{\alpha \in \Pi-\Pi_{0}} \boldsymbol{R}^{+} \Lambda_{\alpha}
$$

in a natural way, and leaves invariant the discrete semigroup

$$
Z_{\mathrm{c}}^{+}=\sum_{\alpha \in \Pi-\Pi_{0}} Z^{+} \Lambda_{\alpha}
$$

in $\mathrm{c}^{+}$.

1) Let $\mathscr{K}(M)$ be the set of homogeneous Kähler structures on $M$. Then the correspondence $\lambda \mapsto(M, g(\lambda)), \lambda \in \mathfrak{c}^{+}$, induces a bijection from the orbit space Aut $\left(\Pi, \Pi_{0}\right) \backslash c^{+}$onto the set $\mathscr{K}(M)$.

2) Let $\mathscr{K}_{0}(M)$ be the subset of $\mathscr{K}(M)$ consisting of all homogeneous Kähler structures on $M$ admitting Kähler immersions into Fubini-Study 
spaces (of constant holomorphic sectional curvatures c). Then the correspondence $\lambda \mapsto\left(M, \frac{8 \pi}{c} g(\lambda)\right)$ induces a bijection from the orbit space Aut $\left(\Pi, \Pi_{0}\right) \backslash Z_{\mathrm{c}}^{+}$onto the set $\mathscr{H}_{0}(M)$.

3) Let $\mathscr{E}(M)$ be the subset of $\mathscr{K}(M)$ consisting of all homogeneous Kähler Einstein structures on $M$. Then the correspondence $\lambda \mapsto(M, g(\lambda)$ ) induces a bijection from $\boldsymbol{R}^{+} \delta_{\mathrm{m}}$ onto the set $\mathscr{E}(M)$.

4) Let $\mathscr{E}_{0}(M)=\mathscr{K}_{0}(M) \cap \mathscr{E}(M)$, i.e., $\mathscr{E}_{0}(M)$ be the set of homogeneous Kähler Einstein structures on $M$ admitting Kähler immersions into Fubinistudy spaces. Then the correspondence $\lambda \mapsto\left(M, \frac{8 \pi}{c} g(\lambda)\right)$ induces a bijection from $Z^{+}\left(\sum_{\alpha \in \Pi=\Pi_{0}} \kappa_{\alpha}(M) \Lambda_{\alpha}\right)$ onto the set $\mathscr{E}_{0}(M)$.

Proof. 1) Let $g^{\prime}$ be a Kähler metric on $M$ such that $G^{\prime}=\operatorname{Aut}^{0}\left(M, g^{\prime}\right)$ acts transitively on $M$. Since both $G$ and $G^{\prime}$ are maximal compact subgroups of $G^{c}$, by Theorem 2.1, we have an element $x \in G^{c}$ such that $x G^{\prime} x^{-1}$ $=G$. If we put $g^{\prime \prime}=x \cdot g^{\prime}$, where the dot . means the natural action of Aut $(M)$ on the tensor fields on $M$, then $g^{\prime \prime}$ is a $G$-invariant Kähler metric on $M$ and defines on $M$ the same homogeneous Kähler structures as $g^{\prime}$. Thus, by Theorem 3.1, we may consider only Kähler metrics on $M$ of the forms $g(\lambda)$ with $\lambda \in \mathfrak{c}^{+}$. Note that $\operatorname{Aut}^{0}(M, g(\lambda))=G$ for each $\lambda \in \mathfrak{c}^{+}$. Note further that if $\psi$ is an automorphism of $g^{c}$ with

$$
\psi \mathrm{g}=\mathrm{g}, \quad \psi \mathfrak{u}=\mathfrak{t}, \quad \psi \mathrm{t}=\mathrm{t}, \quad \psi \Pi=\Pi \quad \text { and } \quad \psi \Pi_{0}=\Pi_{0},
$$

then $\psi$, restricted on $\sqrt{-1} t$, is an element of Aut $\left(\Pi, \Pi_{0}\right)$ and it induces in a natural way a holomorphism of $M$, denoted also by $\psi$, satisfying

$$
g(\psi \lambda)=\psi \cdot g(\lambda) \quad \text { for } \lambda \in \mathfrak{c}^{+} .
$$

Now we can prove, is the same way as in Theorem 2.1, the following facts:

(i) For each $\psi \in \operatorname{Aut}\left(\Pi, \Pi_{0}\right)$ with $\psi \lambda=\lambda^{\prime}, \lambda, \lambda^{\prime} \in \mathfrak{c}^{+}$, there exists an automorphism of $\mathrm{g}^{c}$ which extends $\psi$, denoted also by $\psi$, such that it satisfies (3.7) and induces a holomorphism $\psi$ of $M$ satisfying $\psi \cdot g(\lambda)=g\left(\lambda^{\prime}\right)$.

(ii) If there exists a holomorphism $\varphi$ of $M$ with $\varphi \cdot g(\lambda)=g\left(\lambda^{\prime}\right), \lambda, \lambda^{\prime} \in \mathfrak{c}^{+}$, then there exists $\psi \in \operatorname{Aut}\left(\Pi, \Pi_{0}\right)$ satisfying $\psi \lambda=\lambda^{\prime}$.

Therefore, the correspondences $\lambda \mapsto(M, g(\lambda))$ and $(M, g(\lambda)) \mapsto \lambda$ induce the maps Aut $\left(\Pi, \Pi_{0}\right) \backslash \mathfrak{c}^{+} \rightarrow \mathscr{K}(M)$ and $\mathscr{K}(M) \rightarrow$ Aut $\left(\Pi, \Pi_{0}\right) \backslash \mathfrak{c}^{+}$respectively. This implies the assertion 1$)$.

2) follows from the above 1), Theorems 2.2 and 3.2.

3) follows from 1) and Corollary of Theorem 3.1. 
4) follows from 1) and Corollary of Theorem 3.2.

q.e.d.

We get the following corollary from the Theorem, 2), 4) and Corollary 2 of Theorem C.

COROLLARY. Let $M$ be as in the Theorem. Then the set of equivalence classes of full Kähler immersions into Fubini-Study spaces of homogeneous Kähler manifolds (resp. of homogeneous Kähler Einstein manifolds) with the underlying complex manifold $M$, corresponds one to one to the orbit space Aut $\left(I, \Pi_{0}\right) \backslash Z_{\mathrm{c}}^{+}$(resp. to the semigroup $Z^{+}$of positive integers).

REMARK 3.2. Actually, the set $\mathscr{K}(M)$ coincides with the set of Kähler structures on $M$ of constant scalar curvatures, and hence $\mathscr{E}(M)$ with the set of Kähler Einstein structures on $M$ (Matsushima [13]). In fact, let $g$ be a Kähler metric on $M$ of constant scalar curvature. Then, in the same way as the proof of Corollary of Theorem $M$, we conclude that $\operatorname{Aut}^{0}(M, g)$ is a maximal compact subgroup of $\operatorname{Aut}^{0}(M)$ and hence it is transitive on $M$. Thus $g$ is a homogeneous Kähler metric on $M$. This proves the assertion.

\section{§4. Locally symmetric Kähler submanifolds of $\boldsymbol{P}_{N}(c)$}

In this section we shall classify symmetric Kähler submanifolds of $P_{N}(c)$ and study certain geometric invariants of them. As an application we shall reproduce Nakagawa-Takagi's classification theorem for Kähler submanifolds with parallel second fundamental forms.

THEOREM 4.1. 1) Let a locally symmetric Kähler manifold $(M, g)$ admit a Kähler immersion into a Fubini-Study space. Then $(M, g)$ is locally of compact type.

2) Let a complete locally symmetric Kähler manifold $(M, g)$ admit a Kähler immersion into a Fubini-study space. Then $(M, g)$ is a (global) symmetric Kähler manifold of compact type.

3) Let $(M, g)$ be a symmetric Kähler manifold of compact type and $U$ an open connected subset of $M$. Then, each local Kähler immersion: $(U, g) \rightarrow P_{N}(c)$ is extended uniquely to a global Kähler immersion: $(M, g) \rightarrow$ $P_{N}(c)$.

Proof. 1) Let $f:(M, g) \rightarrow P_{N}(c)$ be a Kähler immersion. Take an open connected subset $U$ of $M$ such that $(U, g)$ is an open Kähler submanifold of a simply connected symmetric Kähler manifold $(\bar{M}, \bar{g})$. Then, by Theorem C (II), there exists uniquely a Kähler immersion $\bar{f}:(\bar{M}, \bar{g}) \rightarrow P_{N}(c)$ such that $\bar{f}|U=f| U$. By Theorem 2.1, $(\bar{M}, \bar{g})$ is then of compact type. This proves the assertion 1). 2) is an immediate consequence of 1) and 
Corollary of Theorem 2.1. 3) follows from Theorem C (II).

q.e.d.

So we shall study in detail Kähler immersions of symmetric Kähler manifolds into $P_{N}(c)$. We retain the notation in the previous sections.

Let $\Pi=\left\{\alpha_{1}, \cdots, \alpha_{l}\right\}$ be an irreducible Dynkin diagram and $\Pi_{0}$ a subset of $\Pi$ such that $\Pi-\Pi_{0}$ consists of only one root, say $\alpha_{1}$, and that the highest root $\alpha$ of the root system $\Sigma$ with the fundamental root system $\Pi$ has an expression :

$$
\alpha=\alpha_{1}+\sum_{i=2}^{l} m_{i} \alpha_{i}, \quad m_{i} \in Z
$$

Such a pair $\left(\Pi, \Pi_{0}\right)$ is said to be irreducible symmetric. Let now $\left(\Pi, \Pi_{0}\right)$ be a general pair of Dynkin diagrams. Decompose $\Pi$ into the sum of irreducible components :

$$
\Pi=\Pi^{1} \cup \cdots \cup \Pi^{s},
$$

and put

$$
\Pi_{0}^{i}=\Pi^{i} \cap \Pi_{0} \quad(1 \leqslant i \leqslant s) .
$$

If each pair $\left(\Pi^{i}, \Pi_{0}^{i}\right)$ is irreducible symmetric, the pair $\left(\Pi, \Pi_{0}\right)$ is said to be symmetric. Note that a symmetric pair $\left(\Pi, \Pi_{0}\right)$ is always effective. Then the following results are known (cf. Takeuchi [18], Helgason [7]).

THEOREM 4.2. Let $M=G / K$ be the homogeneous complex manifold associated to an effective pair $\left(\Pi, \Pi_{0}\right)$ of Dynkin diagrams. Then, the pair $(G, K)$ is a symmetric pair if and only if $\left(\Pi, \Pi_{0}\right)$ is symmetric. In this case, for any G-invariant Kähler metric $g$ on $M,(M, g)$ is a symmetric Kähler manifold and $\operatorname{Aut}^{0}(M, g)=G$. Any symmetric Kähler manifold of compact type is obtained in this way.

For a symmetric pair $\left(\Pi, \Pi_{0}\right)$, the rank of the associated symmetric Kähler manifold $M=G / K$ will be called the rank of $\left(\Pi, \Pi_{0}\right)$.

REMARK 4.1. Let $(M, g)$ be a symmetric Kähler manifold of compact type associated to a symmetric pair $\left(\Pi, \Pi_{0}\right)$. Then the Ricci tensor $S$ for $g$ is given by

$$
S=\sum_{\alpha \in \Sigma_{\mathrm{m}}^{+}} \omega^{-\alpha} \cdot \bar{\omega}^{-\alpha}
$$

This follows from a formula:

$$
S(X, Y)=-\frac{1}{2}(X, Y) \quad \text { for } X, Y \in \mathfrak{m}=T_{o} M
$$


for a general symmetric Riemannian manifold (Takeuchi-Kobayashi [19]).

COROLLARY. The set of equivalence classes of admissible triples $\left(\Pi, \Pi_{0} ; p\right)$ with $\left(\Pi, \Pi_{0}\right)$ symmetric is in a bijective correspondence with the set of equivalence classes of full Kähler immersions of symmetric Kähler manifolds into Fubini-Study spaces (of constant holomorphic sectional curvatures $c$ ).

Proof. Let $\left(\Pi, \Pi_{0} ; p\right)$ be an admissible triple with $\left(\Pi, \Pi_{0}\right)$ symmetric and $f:(M, g) \rightarrow P_{N}(c)$ the full Kähler immersion of a symmetric Kähler manifold $(M, g), M=G / K$, associated to $\left(\Pi, \Pi_{0} ; p\right)$. Then, since we have $G=\operatorname{Aut}^{0}(M, g)$ by Theorem 4.2, the admissible triple associated to $f$ is equivalent to $\left(\Pi, \Pi_{0} ; p\right)$. Thus, in virtue of Lemma 2.4 , the correspondence in Theorem 2.2 is injective on the set of all equivalence classes of admissible triples $\left(\Pi, \Pi_{0} ; p\right)$ with $\left(\Pi, \Pi_{0}\right)$ symmetric. This, together with Theorem 2.2 , implies the Corollary.

LEMMA 4.1. Let $\left(\Pi, \Pi_{0}\right)$ be an irreducible symmetric pair with $\Pi-\Pi_{0}$ $=\left\{\alpha_{1}\right\}$, and $M$ the homogeneous complex manifold associated to $\left(\Pi, \Pi_{0}\right)$. Put

$$
q(M)=\sharp\left\{\alpha \in \Sigma_{\mathfrak{m}}^{+} ;\left(\alpha, \alpha_{1}\right)>0\right\},
$$

and

$$
\nu(M)=\sharp\left\{\alpha \in \Sigma_{\mathfrak{m}}^{+}-\left\{\alpha_{1}\right\} ; \alpha-\alpha_{1} \in \Sigma\right\} .
$$

Then the integer $\kappa(M)$ defined in $\S 3$ is given by

$$
\kappa(M)=q(M)+1=\nu(M)+2 .
$$

Proof. Since each $\alpha \in \Sigma_{\mathrm{m}}^{+}$has also an expression as (4.1), we have

$$
\alpha-2 \alpha_{1} \notin \Sigma, \quad \alpha+\alpha_{1} \notin \Sigma \quad \text { for } \alpha \in \Sigma_{\mathfrak{m}}^{+}-\left\{\alpha_{1}\right\} .
$$

Hence, for $\alpha \in \Sigma_{\mathrm{m}}^{+}-\left\{\alpha_{1}\right\}$, we have

$$
\frac{2\left(\alpha, \alpha_{1}\right)}{\left(\alpha_{1}, \alpha_{1}\right)}= \begin{cases}0 & \text { if } \alpha-\alpha_{1} \oplus \Sigma \\ 1 & \text { if } \alpha-\alpha_{1} \in \Sigma .\end{cases}
$$

This implies $q(M)=\nu(M)+1$. Recalling that in our case

$$
\kappa(M)=\sum_{\alpha \in \Sigma_{\mathrm{m}}^{+}} \frac{2\left(\alpha, \alpha_{1}\right)}{\left(\alpha_{1}, \alpha_{1}\right)},
$$

we get also $\kappa(M)=q(M)+1$.

q.e.d.

Let $\left(\Pi, \Pi_{0} ; p\right)$ be an admissible triple such that $\left(\Pi, \Pi_{0}\right)$ is irreducible 
symmetric and let $\Pi-\Pi_{0}=\left\{\alpha_{1}\right\}$. Then the map $p: \Pi-\Pi_{0} \rightarrow Z^{+}$is determined by the positive integer $p_{1}=p\left(\alpha_{1}\right)$ alone. So, in this case, we shall use the notation $\left(\Pi, \Pi_{0} ; p_{1}\right)$ instead of $\left(\Pi, \Pi_{0} ; p\right)$ and call the corresponding immersion $f:(M, g) \rightarrow P_{N}(c)$ the $p_{1}$-th full Kähler immersion of $(M, g)$. With these understood, we have

THEOREM 4.3. Let $\left(\Pi, \Pi_{0} ; p\right)$ be an admissible triple with $\left(\Pi, \Pi_{0}\right)$ irreducible symmetric, $p \in Z^{+}$, and let $f:(M, g) \rightarrow P_{N}(c)$ be the corresponding $p$-th full Kähler immersion of irreducible symmetric Kähler manifold $(M, g)$. Then

$$
\begin{aligned}
& g=\frac{2 p}{c(q(M)+1)} \sum_{\alpha \in \Sigma_{\mathfrak{m}}^{+}} \omega^{-\alpha} \cdot \bar{\omega}^{-\alpha}, \\
& S=\frac{c(q(M)+1)}{2 p} g, \\
& k=\frac{n c(q(M)+1)}{p} .
\end{aligned}
$$

Hence, in this case, for a given $M$ the Kähler structure of $(M, g)$ is determined by the scalar curvature $k$.

Proof. The formulas for $S$ and $k$ follow from Corollary of Theorem 3.2 and Lemma 4.1. The formula for $g$ follows from Remark 4.1 and the formula for $S$.

q.e.d.

REMARK 4.2. The formula for $k$ was also obtained by Nakagawa-Takagi [15] in a different way from ours.

Comparing $g$ above and $g=\frac{p}{c} g_{M}$ in Corollary of Theorem 3.2, we get

$$
4\left(\delta_{\mathfrak{m}}, \alpha\right)=1 \quad \text { for } \alpha \in \Sigma_{\mathfrak{m}}^{+} .
$$

This and $\kappa(M)=4\left(\delta_{\mathfrak{m}}, \alpha_{1}\right) /\left(\alpha_{1}, \alpha_{1}\right)$ imply

$$
\kappa(M)\left(\alpha_{1}, \alpha_{1}\right)=(q(M)+1)\left(\alpha_{1}, \alpha_{1}\right)=1 . \quad \text { (Murakami [14]) }
$$

Hence, denoting the Kähler metric defined by - (, ) by

$$
g_{0}=2 \sum_{\alpha \in \Sigma_{\mathrm{m}}^{+}} \omega^{-\alpha} \cdot \bar{\omega}^{-\alpha},
$$

we get

$$
g=\frac{p}{c(q(M)+1)} g_{0}=\frac{p\left(\alpha_{1}, \alpha_{1}\right)}{c} g_{0} . \quad \text { (Nakagawa-Takagi [15]) }
$$


We give here the table of irreducible symmetric Kähler manifolds of compact type and some of their invariants.

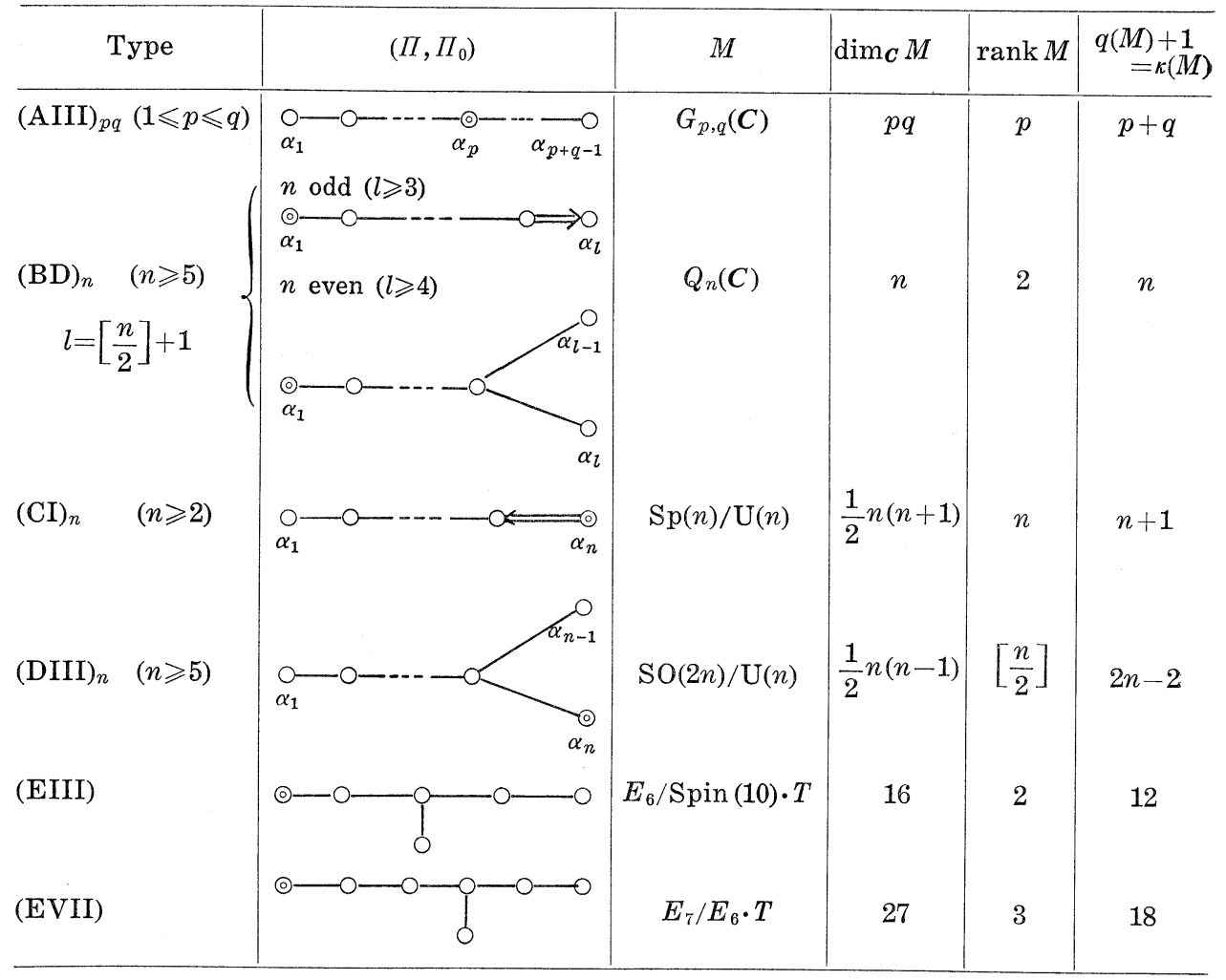

In the table;

( ) represents the element of $\Pi-\Pi_{0}$

$G_{p, q}(C)$ : complex Grassmann manifold of all $p$-dimensional subspaces of $C^{p+q}$ $Q_{n}(C)$ : complex quadric of dimension $n$

$E_{6}, E_{7}: \quad$ compact simply connected Lie groups of type $E_{6}, E_{7}$ respectively

Now we recall the notion of the degrees of Kähler immersions. Let $f:(M, g) \rightarrow P_{N}(c)$ be a Kähler immersion of a Kähler manifold $(M, g)$. The complexification $(T M)^{C}$ of the tangent bundle $T M$ of $M$ has a Whitney-sum decomposition :

$$
(T M)^{c}=T^{+} M \oplus T^{-} M,
$$

where the fibre $T_{p}^{ \pm} M$ over $p \in M$ of $T^{ \pm} M$ is the $\pm \sqrt{-1}$-eigenspace of $(C$ linear extension of) the complex structure tensor $J_{p}$ on $T_{p} M$. In the same way, we get a decomposition:

$$
(N M)^{C}=N^{+} M \oplus N^{-} M
$$


of the complexification $(N M)^{C}$ of the normal bundle $N M$ of $f$. We shall define the higher fundamental form $H_{m}(m \geqslant 2)$ of $f$ as a smooth section of the complex vector bundle Hom $\left(\otimes^{m} T^{+} M, N^{+} M\right)$. In what follows, $C^{\infty}(E)$, for a smooth vector bundle $E$, stands for the space of smooth sections of $E$.

Let $h_{2} \in C^{\infty}\left(\operatorname{Hom}\left(\otimes^{2} T M, N M\right)\right)$ be the second fundamental form of $f$. We define $h_{m} \in C^{\infty}\left(\right.$ Hom $\left.\left(\otimes^{m} T M, N M\right)\right)(m \geqslant 3)$ inductively by

$$
h_{m+1}=\mathscr{D} h_{m},
$$

where $\mathscr{D}: C^{\infty}\left(\operatorname{Hom}\left(\otimes^{m} T M, N M\right)\right) \rightarrow C^{\infty}\left(\operatorname{Hom}\left(\otimes^{m+1} T M, N M\right)\right)$ is the covariant derivation associated to the isometric immersion $f$, i.e.,

$$
\begin{aligned}
& \left(\mathscr{D} h_{m}\right)\left(x_{1} \otimes \cdots \otimes x_{m} \otimes x_{m+1}\right)=D_{x_{m+1}} h_{m}\left(X_{1} \otimes \cdots \otimes X_{m}\right) \\
& \quad-\sum_{i} h_{m}\left(x_{1} \otimes \cdots \otimes \nabla_{x_{m+1}} X_{i} \otimes \cdots \otimes x_{m}\right) \quad \text { for } x_{i} \in T_{p} M,
\end{aligned}
$$

where $X_{i}$ is a smooth local vector field on $M$ around $p$ with $\left(X_{i}\right)_{p}=x_{i}$, and $D$ and $\nabla$ are the normal connection on $N M$ and the Riemannian connection on $T M$ respectively (cf. Kobayashi-Nomizu [10], Chap. VII). Extend $h_{m} C$ bilinearly to a smooth section of $\mathrm{Hom}\left(\otimes^{m}(T M)^{C},(N M)^{C}\right)$, and denote it by the same symbol $h_{m}$. Then

$$
h_{m}\left(x_{1} \otimes \cdots \otimes x_{m}\right) \in N_{p}^{+} M \quad \text { for } x_{i} \in T_{p}^{+} M,
$$

since $h_{2}\left(x_{1} \otimes x_{2}\right) \in N_{p}^{+} M$ for $x_{1}, x_{2} \in T_{p}^{+} M$ (cf. Kobayashi-Nomizu [10], Chap. IX). Now we define $H_{m} \in C^{\infty}\left(\operatorname{Hom}\left(\otimes^{m} T^{+} M, N^{+} M\right)\right)$ by

$$
H_{m}\left(x_{1} \otimes \cdots \otimes x_{m}\right)=h_{m}\left(x_{1} \otimes \cdots \otimes x_{m}\right) \quad \text { for } x_{i} \in T_{p}^{+} M
$$

For $m \in Z^{+}$and $p \in M$, we define a subspace $H_{p}^{m}(M)$ of $T_{f(p)}^{+} P_{N}(C)$ to be the subspace spanned over $C$ by $T_{p}^{+} M$ and the images $H_{k}\left(\otimes^{k} T_{p}^{+} M\right), 2 \leqslant k \leqslant m$. For $m \in Z^{+}$, we define a subset $\mathscr{R}_{m}$ of $M$ inductively as follows. Define $\mathscr{R}_{1}$ $=M$. For $m \geqslant 2$, assume $\mathscr{R}_{m-1}$ is already defined. We then define

$$
\mathscr{R}_{m}=\left\{p \in \mathscr{R}_{m-1} ; \operatorname{dim}_{C} H_{p}^{m}(M)=\max _{p^{\prime} \in \mathfrak{x}_{m-1}} \operatorname{dim}_{C} H_{p^{\prime}}^{m}(M)\right\} .
$$

Then it can be shown (Takagi-Takeuchi [17]) that there exists a unique positive integer $d$ such that

$$
H_{p}^{d-1}(M) \subsetneq H_{p}^{d}(M) \quad \text { for some } p \in \mathscr{R}_{d},
$$

and

$$
H_{p}^{d}(M)=H_{p}^{d+1}(M)=H_{p}^{d+2}(M)=\cdots \quad \text { for each } p \in \mathscr{R}_{d},
$$

where $H_{p}^{0}(M)$ is understood always as $\{0\}$. Such integer $d$ is called the $d e$ - 
gree of $f$ and denoted by $d(f)$. The degree $d(f)$ is related with the number $N(f)$ defined in $\S 1$ as follows.

THEOREM 4.4 (Takagi-Takeuchi [17]). Let $f:(M, g) \rightarrow P_{N}(c)$ be a Kähler immersion of a Kähler manifold $(M, g)$. Then

$$
N(f)=\operatorname{dim}_{C} H_{p}^{d(f)}(M) \quad \text { for } p \in \mathscr{R}_{d(f)} .
$$

For a Kähler immersion of a symmetric Kähler manifold, TakagiTakeuchi computed the degree of it from the corresponding admissible triple.

THEOREM 4.5 (Takagi-Takeuchi [17]). Let $f:(M, g) \rightarrow P_{N}(c)$ be a full Kähler immersion of a symmetric Kähler manifold $(M, g)$. Assume the corresponding admissible triple $\left(\Pi, \Pi_{0} ; p\right)$ has the direct product decomposition:

$$
\left(\Pi, \Pi_{0} ; p\right)=\left(\Pi^{1}, \Pi_{0}^{1} ; p_{1}\right) \times \cdots \times\left(\Pi^{s}, \Pi_{0}^{s} ; p_{s}\right),
$$

with $\Pi^{i}$ irreducible, $p_{i} \in Z^{+}$and rank $\left(\Pi^{i}, \Pi_{0}^{i}\right)=r_{i}(1 \leqslant i \leqslant s)$. Then the degree $d(f)$ of $f$ is given by

$$
d(f)=\sum_{i=1}^{s} r_{i} p_{i}
$$

As an application, we have the following

THEOREM 4.6 (Nakagawa-Takagi [15]). Let $f:(M, g) \rightarrow P_{N}(c)$ be a full Kähler immersion of a Kähler manifold $(M, g)$ with the parallel second fundamental form $h: \mathscr{D} h=0$. Then, $(M, g)$ is locally symmetric and $f$ is locally equivalent to one of the following Kähler immersions of symmetric Kähler manifolds:

1) $(M, g)=P_{n}(c), n \geqslant 1 . \quad f$ is the identity $\operatorname{map}: P_{n}(c) \rightarrow P_{n}(c)$.

2) $(M, g)=P_{n}\left(\frac{c}{2}\right), n \geqslant 1 . \quad f$ is the Kähler imbedding $f: P_{n}\left(\frac{c}{2}\right) \rightarrow$ $P_{(n+2)(n+1) / 2}(c)$ in Example 2.1, with $p=2$.

3) $(M, g)=Q_{n}(C), n \geqslant 3$, with $k=n^{2} c . \quad f$ is the first full Kähler immersion of $(M, g)$. It is induced from the natural map $Q_{n}(C) \longmapsto$ $P_{n+1}(C)$.

4) $(M, g)=G_{2, q}(C), q \geqslant 3$, with $k=2 q(q+2) c$. $f$ is the first full Kähler immersion of $(M, g)$. It is induced from the Plücker map $G_{2, q}(C)$ $\rightarrow P_{(q+2)(q+1) / 2-1}(C)$.

5) $(M, g)=\mathrm{SO}(10) / \mathrm{U}(5)$ with $k=80 c . \quad N+1=16 . \quad f$ is the first full Kähler immersion of $(M, g)$. 
6) $(M, g)=E_{6} / \operatorname{Spin}(10) \cdot T$ with $k=192 c . \quad N+1=27 . \quad f$ is the first full Kähler immersion of $(M, g)$.

7) $(M, g)=P_{n_{1}}(c) \times P_{n_{2}}(c)\left(n_{2} \geqslant n_{1} \geqslant 1\right) . \quad N+1=\left(n_{1}+1\right)\left(n_{1}+2\right) . \quad f$ is the tensor product in Example 2.2.

Proof. Extending the Kähler metric $g_{c}$ of $P_{N}(c) C$-bilinearly to $\left(T P_{N}(C)\right)^{c}$, we define a hermitian inner product $\langle$,$\rangle on \left(T P_{N}(C)\right)^{c}$ by

$$
\langle x, y\rangle=g_{c}(x, \bar{y}) \quad \text { for } x, y \in\left(T_{p} P_{N}(C)\right)^{c} .
$$

Then the Gauss equation for $f$ (cf. Kobayashi-Nomizu [10], Chap. VII) implies

$$
\begin{aligned}
\langle R(Z, \bar{W}) Y, X\rangle= & \frac{c}{2}\{\langle Y, X\rangle\langle Z, W\rangle+\langle Z, X\rangle\langle Y, w\rangle\} \\
& -\langle h(Y \otimes Z), h(X \otimes W)\rangle \quad \text { for } X, Y, Z, W \in C^{\infty}\left(T^{+} M\right),
\end{aligned}
$$

where $R$ denotes the $C$-trilinear extension of the curvature tensor of $(M, g)$. Differentiate (4.2) by a smooth vector field $V$ on $M$. Then, making use of the assumption: $\mathscr{D} h=0$, i.e.,

$$
D_{X}(h(Y \otimes Z))=h\left(\nabla_{X} Y \otimes Z\right)+h\left(Y \otimes \nabla_{X} Z\right)
$$

for every smooth vector fields $X, Y, Z$ on $M$, we get

$$
\left\langle\left(\nabla_{V} R\right)(Z, \bar{W})(Y), X\right\rangle=0 \text {. }
$$

This implies $\nabla R=0$, i.e., $(M, g)$ is locally symmetric. It is known (Nakagawa-Takagi [15]) that if $(M, g)$ is a locally symmetric Kähler manifold, then

$$
\left\langle H_{m}\left(\otimes^{m} T_{p}^{+} M\right), H_{m^{\prime}}\left(\otimes^{m^{\prime}} T_{p}^{+} M\right)\right\rangle=\{0\} \quad \text { for } m \neq m^{\prime},
$$

and that for any Kähler immersion $f:(M, g) \rightarrow P_{N}(c)$

$$
h_{3}(X, Y, \bar{Z})=(\mathscr{D} h)(X, Y, \bar{Z})=0 \quad \text { for } X, Y, Z \in C^{\infty}\left(T^{+} M\right) .
$$

It follows from (4.3) and (4.4) that for a Kähler immersion $f:(M, g) \rightarrow P_{N}(c)$ of a locally symmetric Kähler manifold $(M, g)$, the condition $\mathscr{D} h=0$ is equivalent to $d(f) \leqslant 2$. Thus, by Theorem $4.1,3$ ) and Theorem 4.5, the proof of the Theorem will be completed if we pick up admissible triples $\left(\Pi, \Pi_{0} ; p\right)$, with $\left(\Pi, \Pi_{0}\right)$ symmetric and $\sum_{i=1}^{s} r_{i} p_{i} \leqslant 2$. The possibilities of $s, r_{i}, p_{i}$ are:
a) $s=1, r_{1}=1, p_{1}=1$,
b) $s=1, r_{1}=1, p_{1}=2$,
c) $s=1, r_{1}=2, p_{1}=1$, 
d) $s=2, r_{1}=r_{2}=1, p_{1}=p_{2}=1$.

We get Kähler immersions 1), 2), 3) 6) and 7) according to the cases a), b), c) and d) respectively.

q.e.d.

\section{§5. Nullities of homogeneous Kähler submanifolds of $\boldsymbol{P}_{N}(\boldsymbol{c})$}

In this section, we shall study the nullities as minimal submanifolds (cf. Simons [16]) of homogeneous Kähler submanifolds of $P_{N}(c)$.

We retain the notation in the previous sections. In what follows, for a holomorphic vector bundle $E$ over a complex manifold, $\Gamma(E)$ denotes the space of holomorphic sections of $E$. We need some preliminary lemmas.

Let $E_{N}$ be the canonical line bundle over $P_{N}(C)$ appeared in the proof of Theorem 3.2, and $L_{N}$ the dual line bundle of $E_{N}$. The fibre $\left(L_{N}\right)_{[v]}$ of $L_{N}$ over $[v] \in P_{N}(C), v \in C^{N+1}-\{0\}$, may be considered as the dual space $(C v)^{*}$ of the space $C v$. For each element $\xi$ of the dual space $\left(C^{N+1}\right)^{*}$ of $C^{N+1}$, we define an element $\hat{\xi} \in \Gamma\left(L_{N}\right)$ by

$$
(\hat{\xi}[v])(z v)=\xi(z v) \quad \text { for }[v] \in P_{N}(C), z \in C .
$$

It is not difficult to show that the $\operatorname{map} \xi \mapsto \hat{\xi}$ gives a linear isomorphism: $\left(C^{N+1}\right)^{*} \rightarrow \Gamma\left(L_{N}\right)$, and hence $\operatorname{dim}_{C} \Gamma\left(L_{N}\right)=N+1$.

LEMMA 5.1. Let $f: M \rightarrow P_{N}(C)$ be a full holomorphic immersion of a complex manifold $M$. Then the pull back map $f^{*}: \Gamma\left(L_{N}\right) \rightarrow \Gamma\left(f^{*} L_{N}\right)$ is injective.

Proof. Let $\xi_{0}, \xi_{1}, \cdots, \xi_{N}$ be the standard basis of $\left(C^{N+1}\right)^{*}$. It suffices to show that $f^{*} \hat{\xi}_{0}, f^{*} \hat{\xi}_{1}, \cdots, f^{*} \hat{\xi}_{N}$ are linearly independent. Suppose that $\sum_{i=0}^{N} a_{i} f^{*} \hat{\xi}_{i}=0$ with $a_{i} \in C$. Then, we get

$$
\sum_{i=0}^{N} a_{i} f_{i}(p)=0 \quad \text { for each } p \in M
$$

denoting the homogeneous coordinates of $f(p)$ by $\left(f_{i}(p)\right)_{0 \leqslant i \leqslant N}$. Since $f$ is full, we can find $p_{0}, p_{1}, \cdots, p_{N} \in M$ such that $\operatorname{det}\left(f_{i}\left(p_{j}\right)\right)_{0 \leqslant i, j \leqslant n} \neq 0$, and hence each $a_{j}=0$. This completes the proof.

q.e.d.

Let $f: M \rightarrow P_{N}(C)$ be a full holomorphic immersion of a compact complex manifold $M . \quad f$ is said to be strongly full if the pull back map $f^{*}: \Gamma\left(L_{N}\right) \rightarrow \Gamma\left(f^{*} L_{N}\right)$ is an isomorphism, which is the same as that $\operatorname{dim}_{C} \Gamma\left(f^{*} L_{N}\right)=N+1$, by virtue of the above Lemma.

LEMMA 5.2 (Kimura [8]). Let $f:(M, g) \rightarrow P_{N}(c)$ be a full Kähler immersion of a (compact simply connected) homogeneous Kähler manifold 
$(M, g)$. Then $f$ is strongly full.

Proof. Let $f$ be associated to an admissible triple $\left(\Pi, \Pi_{0} ; p\right)$ and let $\Lambda=\sum_{\alpha \in I I-\Pi_{0}} p_{\alpha} \Lambda_{\alpha}$. Then $N+1$ is equal to the degree of the irreducible representation $\tilde{\rho}$ of $\tilde{G}^{c}$ with the highest weight $\Lambda$. From (3.6), $f^{*} L_{N}$ is the homogeneous line bundle $G^{c} \times_{\tilde{U}} C$ over $M=\tilde{G}^{c} / \tilde{U}$ associated to the character $\chi$ of $\tilde{U}$ satisfying

$$
\chi(\exp H)=e^{-(1, H)} \quad \text { for } H \in t^{C} .
$$

It follows from Borel-Weil theorem (Bott [3]) that $\operatorname{dim}_{C} \Gamma\left(f^{*} L_{N}\right)$ is equal to the degree of the irreducible representation of $\tilde{G}^{c}$ with the lowest weight $-\Lambda$, i.e., to the degree of the representation contragredient to $\tilde{\rho}$. Thus $\operatorname{dim}_{C} \Gamma\left(f^{*} L_{N}\right)=N+1$, which completes the proof.

Let $f: M \rightarrow P_{N}(C)$ be a holomorphic immersion of a complex manifold $M$. The quotient bundle $f^{*} T P_{N}(C) / T M$, a holomorphic vector bundle over $M$, is called the holomorphic normal bunble of $f$ and denoted by $\mathscr{N} M$. If moreover $f:(M, g) \rightarrow P_{N}(c)$ is a Kähler immersion of a Kähler manifold $(M, g)$, then the usual normal bundle $N M$ of $f$ is isomorphic with $\mathscr{N} M$ as a smooth vector bundle in a natural way.

LEMma 5.3. Let $P_{N^{\prime}}(C)$ be an $N^{\prime}$-dimensional linear submanifold of $P_{N}(C)$ and $i: P_{N^{\prime}}(C) \rightarrow P_{N}(C)$ the inclusion map. Let $f: M \rightarrow P_{N}(C)$ and $f^{\prime}: M \rightarrow P_{N^{\prime}}(C)$ be holomorphic immersions of a complex manifold $M$ such that $f=i \circ f^{\prime}$. Let $F^{\prime}$ be the Whitney sum of $\left(N-N^{\prime}\right)$-copies of the line bundle $L_{N^{\prime}}$ over $P_{N^{\prime}}(C)$. Then

1) $f^{*} T P_{N}(C)=f^{\prime *} T P_{N^{\prime}}(C) \oplus f^{\prime *} F^{\prime} \quad$ (holomorphically).

Hence, holomorphic normal bundles $\mathscr{N} M$ and $\mathscr{N}^{\prime} M$ of $f$ and $f^{\prime}$ respectively are related by

$$
\mathscr{N} M=\mathscr{N}^{\prime} M \oplus f^{\prime *} F^{\prime} \quad \text { (holomorphically). }
$$

2) $f^{*} \mathfrak{a}\left(P_{N}(C)\right)=f^{\prime *} \mathfrak{a}\left(P_{N^{\prime}}(C)\right)+f^{\prime *} \Gamma\left(F^{\prime}\right)$ (direct sum).

3) If moreover $f:(M, g) \rightarrow P_{N}(c)$ and $f^{\prime}:(M, g) \rightarrow P_{N^{\prime}}(c)$ are Kähler immersions and if $i: P_{N^{\prime}}(c) \rightarrow P_{N}(c)$ is a totally geodesic imbedding, then the decomposition in 1) is an orthogonal Whitney sum (with respect to the metric $\left.g_{c}\right)$, and

$$
f^{*} \mathrm{~g}\left(P_{N}(c)\right)=f^{*} \mathrm{~g}\left(P_{N^{\prime}}(c)\right)+f^{\prime *} \Gamma\left(F^{\prime}\right) \quad \text { (direct sum). }
$$

Proof. Define holomorphic representations $\lambda_{1}^{*}: \mathrm{SL}(1, N ; C) \rightarrow \mathrm{GL}(1, C)$, $\lambda_{2}: \mathrm{SL}(1, N ; C) \rightarrow \mathrm{GL}(N, C)$ and $\lambda: \operatorname{SL}(1, N ; C) \rightarrow \mathrm{GL}(N, C)$ by 


$$
\begin{aligned}
& \lambda_{1}^{*}:\left(\begin{array}{ll}
\alpha & b \\
0 & d
\end{array}\right) \mapsto \alpha^{-1} \\
& \lambda_{2}:\left(\begin{array}{ll}
\alpha & b \\
0 & d
\end{array}\right) \mapsto d,
\end{aligned}
$$

and

$$
\lambda:\left(\begin{array}{ll}
\alpha & b \\
0 & d
\end{array}\right) \mapsto \alpha^{-1} d
$$

Note that $\lambda$ is nothing but the tensor product $\lambda_{1}^{*} \otimes \lambda_{2}$ of $\lambda_{1}^{*}$ and $\lambda_{2}$. Let $Q_{N}$ be the quotient bundle $1_{N+1} / E_{N}$ of $(N+1)$-dimensional trivial bundle $1_{N+1}$ over $P_{N}(C)$ modulo $E_{N}$. Then, identifying $P_{N}(C)$ with $\operatorname{SL}(N+1, C) / \operatorname{SL}(1, N ; C)$, we get identifications :

$$
\begin{aligned}
L_{N} & =\mathrm{SL}(N+1, C) \underset{\mathrm{SL}(1, N ; C)}{\times} C \text { associated to } \lambda_{1}^{*}, \\
Q_{N} & =\mathrm{SL}(N+1, C) \underset{\mathrm{SL}(1, N ; C)}{\times} C \text { associated to } \lambda_{2}, \\
T P_{N}(C) & =\mathrm{SL}(N+1, C) \underset{\mathrm{SL}(1, N ; C)}{\times} C^{N} \text { associated to } \lambda \\
& =L_{N} \otimes Q_{N} .
\end{aligned}
$$

Each element of $\Gamma\left(T P_{N}(C)\right)=\mathfrak{a}\left(P_{N}(C)\right)$ is identified, as usual, with a holomorphic map $s: \mathrm{SL}(N+1, C) \rightarrow C^{N}$ satisfying

$$
s(x a)=\lambda(a)^{-1} s(x) \quad \text { for } x \in \mathrm{SL}(N+1, C), a \in \mathrm{SL}(1, N ; C) .
$$

For $X \in \mathfrak{g l}(N+1, C)$, we define a holomorphic map $s(X): \operatorname{SL}(N+1, C) \rightarrow C^{N}$ by

$$
x^{-1} X x=\left(\begin{array}{cc}
* & * \\
s(X)(x) & *
\end{array}\right) \quad \text { for } x \in \operatorname{SL}(N+1, C) .
$$

Then $s(X)$ satisfies the above condition and the map $X \mapsto s(X)$ gives an isomorphism $s: \mathfrak{I l}(N+1, C) \rightarrow \mathfrak{a}\left(P_{N}(C)\right)$. In fact, $s$ is nothing but the homomorphism induced from the natural isomorphism: $\mathrm{PL}(N+1, C) \rightarrow$ Aut $\left(P_{N}(C)\right)$. We identify $\operatorname{SL}\left(N^{\prime}+1, C\right)$ with the subgroup

$$
\left\{\left(\begin{array}{cc}
x & 0 \\
0 & 1_{N-N^{\prime}}
\end{array}\right) ; x \in \mathrm{SL}\left(N^{\prime}+1, C\right)\right\}
$$

of SL $(N+1, C)$. We may assume that $i: P_{N^{\prime}}(C) \rightarrow P_{N}(C)$ is the natural inclusion: $\mathrm{SL}\left(N^{\prime}+1, C\right) / \mathrm{SL}\left(1, N^{\prime} ; C\right) \rightarrow \mathrm{SL}(N+1, C) / \mathrm{SL}(1, N ; C)$. Now we are in a position to prove the Lemma. 
1) We have

$$
\begin{aligned}
i^{*} T P_{N}(C) & =i^{*} L_{N} \otimes i^{*} Q_{N}=L_{N} \otimes\left(Q_{N^{\prime}} \oplus 1_{N-N^{\prime}}\right) \\
& =\left(L_{N^{\prime}} \otimes Q_{N^{\prime}}\right) \oplus F^{\prime}=T P_{N^{\prime}} \boldsymbol{C} \oplus F^{\prime},
\end{aligned}
$$

and hence

$$
f^{*} T P_{N}(C)=f^{*} i^{*} T P_{N}(C)=f^{\prime *} T P_{N^{\prime}}(C) \oplus f^{\prime *} F^{\prime}
$$

2) Let

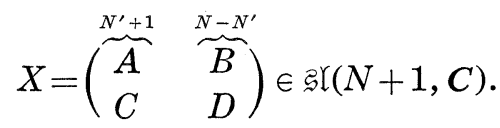

If $X=\left(\begin{array}{ll}0 & B \\ 0 & D\end{array}\right)$, then $s(X)\left(x^{\prime}\right)=0$ for each $x^{\prime} \in \operatorname{SL}\left(N^{\prime}+1, C\right)$, and hence $i^{*} s(X)$ $=0$. If $X=\left(\begin{array}{ll}0 & 0 \\ C & 0\end{array}\right)$, then it induces a holomorphic map $s^{\prime}(X): \operatorname{SL}\left(N^{\prime}+1, C\right)$ $\rightarrow C^{N-N^{\prime}}$ satisfying

$$
\begin{aligned}
s^{\prime}(X)\left(x^{\prime} a^{\prime}\right)=\alpha^{\prime} s^{\prime}(X)\left(x^{\prime}\right) \quad \text { for } x^{\prime} & \in \mathrm{SL}\left(N^{\prime}+1, C\right), \\
a^{\prime} & =\left(\begin{array}{cc}
\alpha^{\prime} & b^{\prime} \\
0 & d^{\prime}
\end{array}\right) \in \mathrm{SL}\left(1, N^{\prime} ; C\right),
\end{aligned}
$$

and hence $i^{*} s(X)$ is an element of $\Gamma\left(F^{\prime}\right)$. Explicitly, $s^{\prime}(X)\left(x^{\prime}\right)$ is the first column vector of the matrix $C x^{\prime}$. It follows that the correspondence $X \mapsto i^{*} s(X)$ is injective. But since the set $\mathfrak{M}^{+}$of such $X$ is a subspace of $\mathfrak{g l}(N+1, C)$ with $\operatorname{dim}_{C} \mathfrak{M}^{+}=\left(N-N^{\prime}\right)\left(N^{\prime}+1\right)=\operatorname{dim}_{C} \Gamma\left(F^{\prime}\right)$, the map $i^{*} \circ s$ induces an isomorphism: $\mathfrak{M}^{+} \rightarrow \Gamma\left(F^{\prime}\right)$. If $X=\left(\begin{array}{cc}A & 0 \\ 0 & 0\end{array}\right) \in \mathfrak{g l}\left(N^{\prime}+1, C\right)$, then $i^{*} s(X) \in$ $\mathfrak{a}\left(P_{N^{\prime}}(C)\right)$. It is easy to see that the map $i^{*} \circ s$ induces also an isomorphism: $\mathfrak{g l}\left(N^{\prime}+1, C\right) \rightarrow \mathfrak{a}\left(P_{N^{\prime}}(C)\right)$. Thus we get

$$
i^{*} \mathfrak{a}\left(P_{N}(C)\right)=\mathfrak{a}\left(P_{N^{\prime}}(C)\right)+\Gamma\left(F^{\prime}\right) \quad \text { (direct sum), }
$$

which implies the assertion 2).

3) Note first that $s: \mathfrak{I l}(N+1, C) \rightarrow \mathfrak{a}\left(P_{N}(C)\right)$ induces an isomorphism $s: \mathfrak{H} \mathfrak{u}(N+1) \rightarrow \mathfrak{g}\left(P_{N}(c)\right)$. In the same way as in 2$)$, we can show that the map $i^{*} \circ s$ induces an $\boldsymbol{R}$-isomorphism from the subspace :

$$
\mathfrak{M}=\left\{\left(\begin{array}{cc}
0 & -{ }^{t} \bar{C} \\
C & 0
\end{array}\right) ; C \text { is an }\left(N-N^{\prime}\right) \times\left(N^{\prime}+1\right) \text {-complex matrix }\right\}
$$

of $\mathfrak{g} \mathfrak{u}(N+1)$ onto $\Gamma\left(F^{\prime}\right)$ as well as an isomorphism: $\mathfrak{H} \mathfrak{u}\left(N^{\prime}+1\right) \rightarrow \mathfrak{g}\left(P_{N}(c)\right)$. Thus we get 


$$
i^{*} \mathrm{~g}\left(P_{N}(c)\right)=\mathrm{g}\left(P_{N^{\prime}}(c)\right)+\Gamma\left(F^{\prime}\right) \quad \text { (direct sum). }
$$

which implies the assertion 3$)$.

q.e.d.

Let $f:(M, g) \rightarrow P_{N}(c)$ be a Kähler immersion of a compact Kähler manifold $(M, g)$. Let $a(N M)$ be the space of all smooth sections $\xi$ of the normal bundle $N M$ of $f$ satisfying

$$
D_{J X} \xi=J D_{X} \xi \quad \text { for each } X \in C^{\infty}(T M),
$$

where $D$ is the normal connection of $N M$ and $J$ is the complex structure tensor of $M$. It is not difficult to see that the natural smooth isomorphism : $N M \rightarrow \mathscr{N} M$ induces an $R$-isomorphism : $\mathfrak{a}(N M) \rightarrow \Gamma(\mathcal{N} M)$. We put

$$
n(f)=\operatorname{dim}_{R} a(N M)=2 \operatorname{dim}_{C} \Gamma(\mathscr{N} M),
$$

and call it the nullity of $f$. Actually, $f$ is always a minimal immersion and $\mathfrak{a}(N M)$ coincides with the space of Jacobi fields along $f$, in the sense of Simons (cf. Simons [16]). Thus $n(f)$ is equal to the dimension of the space of Jacobi fields along $f$.

For $Y \in \Gamma\left(f^{*} T P_{N}(C)\right)$, the normal component of $Y$ with respect to the decomposition: $f^{*} T P_{N}(C)=T M \oplus N M$, will be denoted by $Y^{\perp} \in C^{\infty}(N M)$. We define an $R$-linear map $\nu: a\left(P_{N}(C)\right) \rightarrow C^{\infty}(N M)$ by

$$
\nu(X)=\left(f^{*} X\right)^{\perp} \quad \text { for } X \in \mathfrak{a}\left(P_{N}(C)\right),
$$

and denote the image of the map $\nu$ by $a\left(P_{N}(C)\right)^{\perp}$. It is then known (Simons [16]) that $a\left(P_{N}(C)\right)^{\perp} \subset \mathfrak{a}(N M)$. We put

$$
n_{a}(f)=\operatorname{dim} a\left(P_{N}(C)\right)^{\perp},
$$

and call it the analytic nullity of $f$.

Similarly, the image $\nu\left(\mathfrak{g}\left(P_{N}(c)\right)\right.$ of $g\left(P_{N}(c)\right)$ by the above map $\nu: \mathfrak{a}\left(P_{N}(C)\right)$ $\rightarrow C^{\infty}(N M)$ is denoted by $\mathfrak{g}\left(P_{N}(c)\right)^{\perp}$, which is a subspace of $a\left(P_{N}(C)\right)^{\perp}$. We put

$$
n_{k}(f)=\operatorname{dim} g\left(P_{N}(c)\right)^{\perp},
$$

and call it the Killing nullity of $f$.

From the definitions, we have inequalities:

$$
n(f) \geqslant n_{a}(f) \geqslant n_{k}(f) .
$$

Recall the exact sequences:

$$
0 \longrightarrow \mathfrak{a}\left(P_{N}(C), M\right) \longrightarrow \mathfrak{\longrightarrow}\left(P_{N}(C) \stackrel{\nu}{\longrightarrow} \mathfrak{a}\left(P_{N}(C)\right)^{\perp} \longrightarrow 0,\right.
$$




$$
0 \longrightarrow \mathrm{g}\left(P_{N}(c), M\right) \longrightarrow \mathrm{g}\left(P_{N}(c)\right) \stackrel{\nu}{\longrightarrow} \mathrm{g}\left(P_{N}(c)\right)^{\perp} \longrightarrow 0
$$

to get the equalities:

$$
\begin{aligned}
& n_{a}(f)=2\left\{\operatorname{dim}_{C} \mathfrak{a}\left(P_{N}(C)\right)-\operatorname{dim}_{C} \mathfrak{a}\left(P_{N}(C), M\right)\right\}, \\
& n_{k}(f)=\operatorname{dim} \mathfrak{g}\left(P_{N}(c)\right)-\operatorname{dim} \mathfrak{g}\left(P_{N}(c), M\right) .
\end{aligned}
$$

$f$ is said to be variationally complete if $n(f)=n_{a}(f)$.

THEOREM 5.1. Let $f:(M, g) \rightarrow P_{N}(c)$ be a Kähler immersion of a compact Kähler manifold $(M, g)$. Let $f$ be the composition $i \circ f^{\prime}$ of a full Kähler immersion $f^{\prime}:(M, g) \rightarrow P_{N^{\prime}}(c)$ and a totally geodesic imbedding $i: P_{N}(c) \rightarrow P_{N}(c)$. Then

( I ) $n(f) \geqslant n\left(f^{\prime}\right)+2\left(N-N^{\prime}\right)\left(N^{\prime}+1\right)$; The equality holds if and only if $f^{\prime}$ is strongly full.

( II ) $n_{a}(f)=n_{a}\left(f^{\prime}\right)+2\left(N-N^{\prime}\right)\left(N^{\prime}+1\right)$,

$n_{a}\left(f^{\prime}\right) \geqslant 2\left\{\operatorname{dim}_{C} \mathfrak{a}\left(P_{N^{\prime}}(C)\right)-\operatorname{dim}_{C} \mathfrak{a}(M)\right\} ;$ The equality holds if and only if $f^{\prime}$ is a(M)-equivariant in the sense of $\S 1$.

(III) $n_{k}(f)=n_{k}\left(f^{\prime}\right)+2\left(N-N^{\prime}\right)\left(N^{\prime}+1\right)$, $n_{k}\left(f^{\prime}\right)=\operatorname{dim} g\left(P_{N^{\prime}}(c)\right)-\operatorname{dim} g(M, g)$.

Proof. ( I ) Lemma 5.3, 1) implies

$$
\Gamma(\mathscr{N} M)=\Gamma\left(\mathscr{N}^{\prime} M\right)+\Gamma(f^{\prime *} \underbrace{\left.L_{N^{\prime}}\right)+\cdots+\Gamma\left(f^{\prime *}\right.}_{N-N^{\prime}} L_{N^{\prime}}) \quad \text { (direct sum). }
$$

Now (I) follows from Lemma 5.1.

(II) Lemma 5.3, 2) and 3) imply

$$
\mathfrak{a}\left(P_{N}(C)\right)^{\perp}=\mathfrak{a}\left(P_{N^{\prime}}(C)\right)^{\perp}+f^{\prime *} \Gamma\left(F^{\prime}\right) \quad \text { (direct sum). }
$$

The first equality follows from this and Lemma 5.1. The homomorphism $\sigma^{\prime}: \mathfrak{a}\left(P_{N^{\prime}}(C), M\right) \rightarrow \mathfrak{a}(M)$ for $f^{\prime}$ defined in $\S 1$ is always injective and it is surjective if and only if $f^{\prime}$ is $a(M)$-equivariant. The second assertion follows from this and the equality (5.1) for $f^{\prime}$.

(III) In the same way as the proof of (II), Lemmas 5.3 and 5.1 imply the first equality. The second equality follows from that the homomorphism $\sigma^{\prime}: \mathrm{g}\left(P_{N^{\prime}}(c), M\right) \rightarrow \mathrm{g}(M, g)$ for $f^{\prime}$ is always an isomorphism.

q.e.d.

COROLlaRY 1 (cf. Kimura [8]). Let $f:(M, g) \rightarrow P_{N}(c)$ be a Kähler immersion of a compact Kähler manifold $(M, g)$ with $\operatorname{dim}_{C} M=n$. Then

$$
n(f) \geqslant 2(N-n)(n+1) .
$$


The equality holds if and only if $f$ is totally geodesic. In this case, we have

$$
n(f)=n_{a}(f)=n_{k}(f) .
$$

Proof. Let $f=i \circ f^{\prime}$ be as in the Theorem. Recalling the inequality: $\operatorname{dim} g(M, g) \leqslant n^{2}+2 n$ for a general Kähler manifold $(M, g)$, we have, by the Theorem (III),

$$
\begin{aligned}
n(f)- & 2(N-n)(n+1) \geqslant n_{k}(f)-2(N-n)(n+1) \\
& =n_{k}\left(f^{\prime}\right)+2\left(N-N^{\prime}\right)\left(N^{\prime}+1\right)-2(N-n)(n+1) \\
& =\operatorname{dim} g\left(P_{N^{\prime}}(c)\right)-\operatorname{dim} g(M, g)+2\left(N-N^{\prime}\right)\left(N^{\prime}+1\right)-2(N-n)(n+1) \\
& \geqslant\left(N^{\prime 2}+2 N^{\prime}\right)-\left(n^{2}+2 n\right)+2\left(N-N^{\prime}\right)\left(N^{\prime}+1\right)-2(N-n)(n+1) \\
& =(N+1)\left(N^{\prime}-n\right) \geqslant 0 .
\end{aligned}
$$

The equality holds if and only if $N^{\prime}=n$, i.e., $f$ is totally geodesic. In this case, we have $n(f)=n_{k}(f)$. This completes the proof.

q.e.d.

REMARK 5.1. Actually, Kimura [8] proved the above Corollary in the case where $f$ is an imbedding.

COROLlary 2. Let $f:(M, g) \rightarrow P_{N}(c)$ be a Kähler immersion of a compact Kähler manifold $(M, g)$ of constant scalar curvature. Then

1) If $f$ is full, then

$$
n_{a}(f)=2 n_{k}(f) .
$$

2) $n_{a}(f)=n_{k}(f)$ if only if $f$ is totally geodesic.

Proof. 1) By Lemma 2.1, 3), $f$ is $\mathfrak{a}(M)$-equivariant. It follows from the Theorem (II) that

$$
n_{a}(f)=2\left\{\operatorname{dim}_{C} a\left(P_{N}(C)\right)-\operatorname{dim}_{C} a(M)\right\} .
$$

On the other hand, the Theorem (III) implies

$$
n_{k}(f)=\operatorname{dim} g\left(P_{N}(c)\right)-\operatorname{dim} g(M, g) .
$$

By Lemma 2.1, 2), we have $\operatorname{dim}_{C} \mathfrak{a}(M)=\operatorname{dim} g(M, g)$. This and $\operatorname{dim}_{C}\left(P_{N}(C)\right)$ $=\operatorname{dim} \mathrm{g}\left(P_{N}(c)\right)$ imply the assertion 1$)$.

2) Let $f=f^{\prime} \circ i$ be as in the Theorem. Then, by the Theorem (II), (III) and 1) above,

$$
\begin{aligned}
n_{a}(f) & =n_{k}(f) \Leftrightarrow n_{a}\left(f^{\prime}\right)=n_{k}\left(f^{\prime}\right) \Leftrightarrow 2 n_{k}\left(f^{\prime}\right)=n_{k}\left(f^{\prime}\right) \\
& \Leftrightarrow n_{k}\left(f^{\prime}\right)=0 \Leftrightarrow \operatorname{dim} g\left(P_{N^{\prime}}(c)\right)=\operatorname{dim} g(M, g)
\end{aligned}
$$


THEOREM 5.2. Let $f:(M, g) \rightarrow P_{N}(c)$ be a Kähler immersion of a (compact) homogeneous Kähler manifold $(M, g)$ and let $f=i \circ f^{\prime}$ be as in Theorem 5.1. Then

1) $n(f)=n\left(f^{\prime}\right)+2\left(N-N^{\prime}\right)\left(N^{\prime}+1\right)$.

2) $n_{a}(f)=n_{a}\left(f^{\prime}\right)+2\left(N-N^{\prime}\right)\left(N^{\prime}+1\right)$, $n_{a}\left(f^{\prime}\right)=2 n_{k}\left(f^{\prime}\right)=2\left\{\operatorname{dim}_{C} \mathfrak{a}\left(P_{N^{\prime}}(C)\right)-\operatorname{dim}_{C} \mathfrak{a}(M)\right\}$.

3) $n_{k}(f)=n_{k}\left(f^{\prime}\right)+2\left(N-N^{\prime}\right)\left(N^{\prime}+1\right)$, $n_{k}\left(f^{\prime}\right)=\operatorname{dim} \mathfrak{g}\left(P_{N^{\prime}}(c)\right)-\operatorname{dim} \mathfrak{g}(M, \mathfrak{g})$.

PRoof. 1) follows from Theorem 5.1 (I) and Lemma 5.2.

2) follows from Theorem 5.1 (II) and Corollary 2, 1).

3) follows from Theorem 5.1 (III).

q.e.d.

Recently Kimura proved that a full Kähler imbedding of a compact simply connected homogeneous Kähler manifold is always variationally complete, making use of a theorem of Kostant on Lie algebra cohomology. We give here another proof of this result.

Let $f: M \rightarrow P_{N}(C)$ be a holomorphic immersion of a complex manifold $M$. Denoting by $\pi: \Gamma\left(f^{*} T P_{N}(C)\right) \rightarrow \Gamma(\mathscr{N} M)$ the linear map induced from the natural projection: $f^{*} T P_{N}(C) \rightarrow \mathcal{N} M$, we define a linear map $\nu: \mathfrak{a}\left(P_{N}(C)\right)$ $\rightarrow \Gamma(\mathscr{N} M)$ to be the composition $\pi \circ f^{*}$ of the pull back map $f^{*}$ and $\pi$.

LEMMA 5.4. Let $M$ be a compact complex manifold with $H^{1}(M, \mathcal{O})=\{0\}$ and $H^{1}(M, \Theta)=\{0\}, \mathcal{O}$ (resp. $\Theta$ ) being the sheaf of germs of holomorphic functions (resp. of holomorphic vector fields) on $M$. Let $f: M \rightarrow P_{N}(C)$ be a strongly full holomorphic immersion of $M$ into $P_{N}(C)$. Then the map $\nu$ : $\mathfrak{a}\left(P_{N}(C)\right) \rightarrow \Gamma(\mathcal{N} M)$ is surjective, and $f$ is $\mathfrak{a}(M)$-equivariant.

Proof. We have the following commutative diagram:

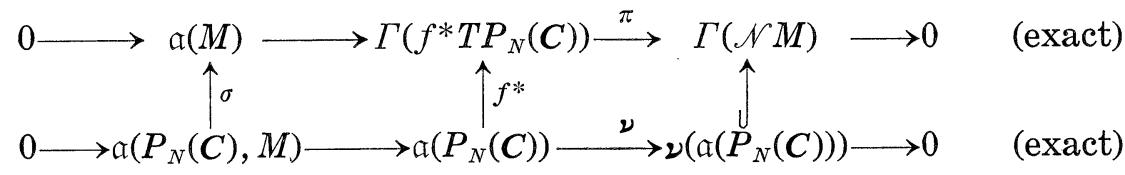

The exactness of the upper row follows from $H^{1}(M, \Theta)=\{0\}$. The fullness of $f$ implies that both $\sigma$ and $f^{*}$ are injective. Therefore, if $\operatorname{dim}_{C} \Gamma\left(f^{*} T P_{N}(C)\right)$ $=\operatorname{dim}_{C} \mathfrak{a}\left(P_{N}(C)\right)=(N+1)^{2}-1$, then $\nu$ is surjective and $f$ is $\mathfrak{a}(M)$-equivariant. Recall now the exact sequence :

$$
0 \rightarrow E_{N} \rightarrow 1_{N+1} \rightarrow Q_{N} \rightarrow 0,
$$

to get an exact sequence : 


$$
0 \rightarrow 1 \rightarrow 1_{N+1} \otimes L_{N} \rightarrow Q_{N} \otimes L_{N} \rightarrow 0 .
$$

Since $T P_{N}(C)=Q_{N} \otimes L_{N}$, we have an exact sequence :

$$
0 \rightarrow 1 \rightarrow \underbrace{L_{N} \oplus \cdots \oplus L_{N}}_{N+1} \rightarrow T P_{N}(C) \rightarrow 0 .
$$

Thus we get an exact sequence :

$$
0 \rightarrow 1 \rightarrow f^{*} \underbrace{L_{N} \oplus \ldots \oplus f^{*}}_{N+1} L_{N} \rightarrow f^{*} T P_{N}(C) \rightarrow 0
$$

of holomorphic vector bundles on $M$. Now $H^{0}(M, \mathcal{O})=C$ and $H^{1}(M, \mathcal{O})=\{0\}$ imply

$$
\operatorname{dim}_{C} \Gamma\left(f^{*} T P_{N}(C)\right)=(N+1) \operatorname{dim}_{C} \Gamma\left(f^{*} L_{N}\right)-1
$$

It follows from the strong fullness of $f$ that $\operatorname{dim}_{C} \Gamma\left(f^{*} T P_{N}(C)\right)=(N+1)^{2}-1$. This completes the proof. q.e.d.

THEOREM 5.3. Let $f:(M, g) \rightarrow P_{N}(c)$ be a Kähler immersion of a homogeneous Kähler manifold $(M, g)$. Then

1) (Kimura) $f$ is variationally complete.

2) Let $f=i \circ f^{\prime}$ be as in Theorem 5.1 and $\left(\Pi, \Pi_{0} ; p\right)$ the admissible triple associated to $f^{\prime}$. Then the nullity $n(f)$ is given by

$$
n(f)=2\left\{(N+1)(N(f)+1)-1-\operatorname{dim}_{C} \mathfrak{a}(M)\right\},
$$

where

$$
N(f)+1=\prod_{\alpha \in \Sigma^{+}} \frac{(\Lambda+\delta, \alpha)}{(\delta, \alpha)}, \quad \Lambda=\sum_{\alpha \in \Pi-\Pi_{0}} p_{\alpha} \Lambda_{\alpha}, \quad \delta=\frac{1}{2} \sum_{\alpha \in \Sigma^{+}} \alpha .
$$

Proof. 1) Theorem $5.2,1$ ) and 2 ) imply that $f$ is variationally complete if and only if $f^{\prime}$ is so. So we may assume that $f$ is full. Now, $b_{1}(M)=0$ implies $H^{1}(M, \mathcal{O})=\{0\}$, and hence a theorem of Bott [3] and Lemma 5.2 imply that our $f$ satisfies the assumptions in Lemma 5.4. Thus the map $\nu: a\left(P_{N}(C)\right) \rightarrow \Gamma(\mathcal{N} M)$ is surjective, which is equivalent to that $\nu: \mathfrak{a}\left(P_{N}(C)\right)$ $\rightarrow \mathfrak{a}(N M)$ is surjective. This means the variationally completeness of $f$.

2) The above 1), Theorem 5.2, 1) and 2) imply

$$
\begin{aligned}
n(f) & =2\left(\operatorname{dim}_{C} P_{N^{\prime}}(C)-\operatorname{dim}_{C} \mathfrak{a}(M)\right)+2\left(N-N^{\prime}\right)\left(N^{\prime}+1\right) \\
& =2\left\{\left(N^{\prime}+1\right)^{2}-1+\left(N-N^{\prime}\right)\left(N^{\prime}+1\right)-\operatorname{dim}_{C} \mathfrak{a}(M)\right\} \\
& =2\left\{(N+1)\left(N^{\prime}+1\right)-1-\operatorname{dim}_{C} \mathfrak{a}(M)\right\} .
\end{aligned}
$$

Here $N^{\prime}=N(f)$ is given by the Weyl formula as in the Theorem, as was 
stated in Remark 2.3. q.e.d. Corollary 2 of Theorem 5.1 and 1) of the Theorem imply the following

COROLlary. Let $f$ be as in the Theorem. Then

1) If $f$ is full, then

$$
n(f)=2 n_{k}(f) .
$$

2) $n(f)=n_{k}(f)$ if and only if $f$ is totally geodesic.

\section{References}

[1] A. Borel and F. Hirzebruch, Characteristic classes and homogeneous spaces I, Amer. J. Math., 80 (1958), 458-538.

[2] A. Borel und R. Remmert, Über kompakte homogene Kählersche Mannigfaltigkeiten, Math. Ann., 145 (1961/1962), 429-439.

[ 3 ] R. Bott, Homogeneous vector bundles, Ann. of Math., 66 (1957), 203-248.

[ 4 ] E. Calabi, Isometric imbeddings of complex manifolds, Ann. of Math., 58 (1953), $1-23$.

[ 5 ] C. Chevalley and S. Eilenberg, Cohomology theory of Lie groups and Lie algebras, Trans. Amer. Math. Soc., 63 (1948), 85-124.

[6] J. Hano and Y. Matsushima, Some studies of Kählerian homogeneous spaces, Nagoya Math. J., 11 (1957), 77-92.

[ 7 ] S. Helgason, Differential Geometry and Symmetric Spaces, Academic Press, New York, 1962.

[ 8 ] Y. Kimura, The nullity of compact Kähler submanifolds in a complex projective space, to appear in J. Math. Soc. Japan.

[ 9 ] S. Kobayashi, Transformation Groups in Differential Geometry, Springer-Verlag, Berlin, 1972.

[10] S. Kobayashi and K. Nomizu, Foundations of Differential Geometry I, II, Interscience, New York, 1963, 1969.

[11] A. Lichnerowicz, Isométries et transformations analytiques d'une variété Kählérienne compacte, Bull. Math. Soc. France, 87 (1959), 427-437.

[12] Y. Matsushima, Sur les espaces homogènes Kählériennes d'un groupe de Lie réductif, Nagoya Math. J., 11 (1957), 53-60.

[13] Y. Matsushima, Remarks on Kähler-Einstein manifolds, Nagoya Math. J., 46 (1972), 161-173.

[14] S. Murakami, Cohomology groups of vector valued forms on symmetric spaces, Lecture Notes Chicago Univ., 1966.

[15] H. Nakagawa and R. Takagi, On locally symmetric Kähler submanifolds in a complex projective space, to appear.

[16] J. Simons, Minimal varieties in Riemannian manifolds, Ann. of Math., 88 (1968), 62-105.

[17] R. Takagi and M. Takeuchi, Degree of symmetric Kählerian submanifolds of a complex projective space, to appear in Osaka J. Math.

[18] M. Takeuchi, Cell decompositions and Morse equalities on certain symmetric spaces, J. Fac. Sci. Univ. Tokyo, Sect. I, 12 (1965), 81-192. 
[19] M. Takeuchi and S. Kobayashi, Minimal imbeddings of $R$-spaces, J. Differential Geometry, 2 (1969), 203-215.

\section{Department OF MATHEMATiCS \\ OSAKA UNIVERSITY \\ TOYONAKA 560}

JAPAN 